\title{
The Communication Infrastructures of Living Systems and the Universe: Analysis from the Perspective of Antenna Theory
}

Sirsendu Sekhar Ray ${ }^{1 *}$, Bitop Maitra ${ }^{1}$

Biomolecular Nanotechnology Laboratory, Department of Biotechnology and Medical Engineering, National Institute of Technology Rourkela, 769008, Odisha, India

${ }^{1}$ Equal authorship

*Corresponding author: Sirsendu Sekhar Ray, sirsendu@nitrkl.ac.in

License information: This work is licensed under CC BY-NC-ND 4.0

\begin{abstract}
It is still unknown how molecules coordinate their activity and operate at high speeds in the crowded environment of a cell. The study focuses on the geometry of biomolecules, assuming B-DNA, $\alpha$-helix, $\beta$-strand, water molecules, and chemical bonds, including hydrogen bonds, as various types of antennas. The analysis demonstrates that living systems have highly sophisticated wireless and wired communication infrastructures for regulating and coordinating molecular activities, revealing why water is essential for molecular dynamics and indicating how we evolved. The study also includes a few equations linking antenna fields with Einstein's general relativity, Kepler's law of planetary motion, and Newton's law of gravitation, which divides the gravitational field into antenna field zones and clarifies many astronomical facts. The findings, furthermore, suggest that the gravitational field is the antenna field of astronomical objects; and that nature's antennas, such as molecules and astronomical objects, communicate via gravitational waves. We hope that the study, which uses a classical approach to explain the facts of living systems and the Universe, will find applications in biology, astronomy, communication engineering, and other areas of science.
\end{abstract}

Keywords: Biology, Astronomy, Wireless communication, Gravitational waves, Antenna field 


\section{Preface}

We had no idea we would come to these conclusions when we started the project. We were surprised by the results as we examined more facts in molecular biology and astronomy from the perspective of antenna theory and radiative field. We really want the article reviewed so that we can figure out where we went wrong. You are welcome to leave a comment or contact us.

This article primarily contains three results, but all of them are controversial and debatable.

\section{Result 1: The living systems have wireless communication among the biomolecules.}

We emphasized the geometry of biomolecules and assumed that B-DNA, $\alpha$-helix, $\beta$-strand, water molecules, and chemical bonds, including hydrogen bonds, operate as antennas and calculated their operating wavelengths and wirelengths. The results show that if B-DNA, $\alpha$-helix, and $\beta$-strand act as antennas, they communicate efficiently (more than the antennas we use). Even the wavelengths of water as a V-shaped antenna communicate efficiently with the B-DNA and $\alpha$-helix. Surprisingly, all the chemical bonds of biomolecules are perfect short dipoles $(\lambda / 10)$ and Hertzian dipoles $(\lambda / 100)$ to the wavelengths of B-DNA and $\alpha$-helix.

a) The analysis indicates how molecules search out another molecule at high speed in a crowded environment.

b) Why there are separate circular DNA inside mitochondria?

c) Why DNA reorients inside a cell?

d) Why are biomolecules predominantly right-handed, and why does denaturation result in function loss?

e) How do the cytoskeletal systems operate as transmission lines?

f) Why is water so important in molecular dynamics?

g) How does the coiling/ uncoiling of DNA regulate transcription?

h) How did we evolve, and what is consciousness?

There are more facts of living systems that are explained in the article. However, the wavelengths of the molecular antennas are in the X-ray and deep-UV range, which is incompatible with living systems. That is why we started working with gravitational waves.

\section{Result 2: Gravitational field is the antenna field (radiative field) of the magnetic dipole of astronomical objects.}

We assumed that the magnetic dipole of the astronomical objects (Sun /Planets) operate as antennas. Furthermore, the event horizon of the general relativity is the aperture of the antenna, and Schwarzschild radius is the operating wavelength of the antenna. After that, we proposed the following equation linking gravitational field and antenna field 


$$
\frac{R^{3}}{T^{2}}=\frac{c^{2} \lambda_{s}}{8 \pi^{2}}=(0.62)^{2} \frac{c^{2}}{8 \pi^{2}} \frac{(D)^{3}}{(\operatorname{ReNFR})^{2}}=\frac{G\left(M_{1}+M_{2}\right)}{4 \pi^{2}}
$$

Where ' $R$ ' represents the semi-major axis of the revolving object, ' $T$ ' represents the orbital period of the revolving object, ' $\lambda_{s}$ ' represents the Schwarzschild radius of the astronomical object, ' $D$ ' represents the length of a magnetic dipole antenna, and 'ReNFR' denotes reactive near field radius of the antenna, ' $c$ ' means the speed of light, ' $M$ ' is the mass of the astronomical object, and ' $G$ ' denotes the Gravitational Constant.

The equation divided the solar system into three zones, reactive near field zone, Fresnel zone, and Fraunhofer's zone, and demonstrated that the asteroid belt, Kuiper's belt, and Oort clouds are caused by scattering. It also explains why Jupiter has decametric emission and why planets within asteroid belts are rocky while planets outside asteroid belts are gaseous and have rings. There are many more facts that are explained in the article. The results indicate that the gravitational field is the antenna field of astronomical objects.

Result 3: if the gravitational field is the antenna field, then the gravitational waves, which are waves over the gravitational field, are the antenna field waves. This establishes the existence of radiating field waves or antenna field waves in the absence of electromagnetic radiation. Therefore, all nature's antennas, including biomolecules, communicate through gravitational waves.

Result 3 is obvious if Result 1 and Result 2 are correct in analyzing the known facts of living systems and the Universe, for which still we do not have many answers. 
The mystery of how molecules communicate to keep living systems running remains unsolved. The conventional and widely accepted theory that has described molecular communication via both the chemical and physical contact modes has generated enormous biological facts that help build up modern biology with immense success. However, the question of how molecules coordinate at high speeds inside cells remains unanswered. $(1,2)$ Furthermore, little is known about how a molecule searches for other molecules in a crowded cell environment for contact mode communication. $(3,4)$ Over the years, various hypotheses for high-speed communication and how molecule searches for another inside a cell have been discussed in the literature, including electromagnetic communication, vibrational resonance, and diffusion theory. (5-9) However, these hypotheses have been unsuccessful in explaining the wellknown biological facts and clarifying the incompatibility of electromagnetic radiation and vibrational resonance with living systems. $(10,11)$ As a result, the puzzle remains open for solutions that will explain high-speed communication and coordination within a cell remaining consistent and compatible with biological facts.

One of the most remarkable technological achievements of the last century has been communication engineering. Antennas, which are various geometries of conductors, are critical in communication engineering because they enable contactless (wireless) communication by transmitting and receiving signals. Biomacromolecules such as DNA and proteins are conductive in living systems and exist in various geometries similar to antennas. (12-18) Because of the similarities between biomacromolecules and antennas, we assumed that biomacromolecules act as antennas and facilitate high-speed wireless communication in crowded cell environments. Therefore, in this study, we emphasized the significance of biomolecular geometry and investigated them from the perspective of antenna theory to explore the possibility of wireless communication in living systems.

The manuscript is divided into four parts. The first part will explain the communication in living systems considering biomolecules as antennas. Part two will describe how communication works in the Universe and why wireless communication in living systems is compatible. After discussing its limitations and implications, we will summarise and conclude the study in the third and fourth parts.

\section{Communication infrastructures of living systems}

Part 1 has four sections. In section one, we assumed that B-DNA and $\alpha$-helix operate as helical antennas and each nucleotide and amino acid are the segments of the helical antennas. (19) (20) We considered only the B-DNA and $\alpha$-helix for the study, as they are the most commonly observed helical structures of the genomes and proteins under physiologic conditions. Section two explains the significance of a $\beta$ strand as zigzag and Vee- antenna. Section three will show that cytoskeletons operate as transmission lines inside a cell. Furthermore, the section will describe histones and spermidines from the antenna theory perspective. Section four will describe water molecules as Vee-antennas and demonstrate that chemical bonds operate as dipole antennas and communicate with the biomacromolecules. 


\subsection{Role of B-DNA and $\alpha$-helix}

In a crowded environment, B-DNA and $\alpha$-helix as helical antennas provide numerous advantages. Helical antennas can track and monitor activity efficiently by emitting a directive signal (for point-topoint communication) in axial mode and an omnidirectional signal (for broad coverage) in normal mode. Furthermore, helical antennas transmit information using circularly polarised waves that increase the likelihood of establishing a reliable connection in a crowded environment. However, in order to receive a circularly polarised wave, the handedness of the receiving antenna must be the same as that of the transmitting antenna; otherwise, the receiving side will suffer a significant loss of gain. The need for similar-handed structures for efficient communication explains why the B-DNA, the $\alpha$-helix, and the twisting of $\beta$-strands are identical right-handed structures.

B-DNA, as bifilar helical antenna, has advantages over $\alpha$-helix, the monofilar helical antenna. A bifilar helical antenna has a broader bandwidth and higher gain, does not need a ground, monitors multiple signals more efficiently, and functions as a highly efficient transceiver system. $(21,22)$ As a result, these additional characteristics suggest that B-DNA functions as an efficient base station or regulator of cellular activities. Furthermore, it appears that the nucleus of a cell rotates, orients, and constantly changes position so that DNA can coordinate and control multiple cellular activities simultaneously.

\subsubsection{Antenna parameters of B-DNA and $\alpha$-helix}

It is difficult to assess biomacromolecules' structural parameters accurately due to conformational change. As a result, we calculated the antenna parameters of B-DNA and $\alpha$-helix using only the dimensions that have been widely reported in the literature. (Table 1) The diameter and pitch of the BDNA are $20 \AA$ and $34 \AA$, whereas the diameter and pitch of $\alpha$-helix are $4.6 \AA$ and $5.4 \AA$ (Figure 1AB). Because a bifilar antenna represents a monofilar antenna with a parasitic helix, we used the formula of a monofilar helix antenna to calculate the parameters of the bifilar helix antenna (B-DNA).

The axial mode wavelength of the helical antenna is equal to the circumference of the helix; therefore, the axial mode wavelengths are $62.83 \AA$ for B-DNA and $14.45 \AA$ for $\alpha$-helix. (SI Eq. 1.1.1). Helical antennas, in normal mode, transmit circularly polarized waves when the axial ratio is one. Therefore, considering the axial ratio as one, the normal mode wavelengths are $58.06 \AA$ for B-DNA and $19.34 \AA$ for $\alpha$-helix (SI Eq. 1.1.2). However, because the axial ratio relates to the number of turns and the normal mode wavelength can be much longer than the antenna's axial mode wavelength, we varied the axial ratio in integers, emphasizing the number of turns. (24) As a result, the additional normal mode wavelengths for the B-DNA are 116.11 $\AA, 174.17 \AA$, and $232.23 \AA$, while the other normal mode wavelengths for the $\alpha$-helix are $38.67 \AA, 58.01 \AA, 77.35 \AA, 96.69 \AA, 116.02 \AA, 135.36 \AA, 154.7 \AA$, 174.03 $\AA, 193.37 \AA, 212.71 \AA, 232.05 \AA$ and $251.38 \AA$. Although the multiples can go much higher 
than the values shown, we kept the multiples within $256 \AA$ for the convenience of the study. Notably, B-DNA and $\alpha$-helix shares the wavelengths $\approx 58 \AA, \approx 116 \AA, \approx 174 \AA$, and $\approx 232 \AA$.

A single turn of B-DNA and $\alpha$-helix has wirelengths of $71.44 \AA$ and $15.43 \AA$, respectively (SI Eq. 1.1.3). The wirelengths of a single turn B-DNA and $\alpha$-helix when operating as electrically small helical antennas, or as small B-DNA and small $\alpha$-helix, are $96.83 \AA$ and $19.85 \AA$, respectively (SI Eq. 1.1.4). However, in order to function as a small helical antenna, the wavelength of the receiving signal must be at least ten times greater than the wire length. This can be accomplished by using a normal mode helical antenna with a higher axial ratio or by incorporating another antenna, which transmits a signal with a longer wavelength. Indeed, the need for a longer wavelength so that DNA can also work as a small helical antenna could be why DNA and plasmids are circular (loop antennas) in prokaryotes and why there is additional circular DNA in the eukaryotic mitochondria. (25)

The self-resonance wavelengths of a single turn of B-DNA and $\alpha$-helix, where the antenna appears purely resistive, are $122.64 \AA$ and $31.69 \AA$, respectively. (SI Eq. 1.1.5). Furthermore, as a half-turn bifilar antenna, another wavelength of B-DNA is the perimeter of the rectangle formed by the wirelength and diameter of the B-DNA (SI Eq. 1.1.6). (26) As a result, another wavelength of the BDNA is $182.88 \AA$.

The pitch angle of B-DNA $\left(28.42^{\circ}\right)$ indicates that B-DNA operates primarily in normal mode, whereas the pitch angle of $\alpha$-helix $\left(20.5^{\circ}\right)$ reveals that $\alpha$-helix communicates through both normal and axial modes (SI Eq. 1.1.7).

Furthermore, using the wirelengths and number of segments per turn (3.6 amino acids per turn of $\alpha$ helix; 10.5 nucleotides per turn of B-DNA), the amino acids lengths are $5.51 \AA$ (19.85 $/ 3.6$ ), and 4.29 $\AA(15.43 \AA / 3.6)$ in $\alpha$-helix and the nucleotides lengths are $9.22 \AA(96.83 \AA / 10.5)$, and $6.8 \AA$ (71.44 $\AA / 10.5)$ in B-DNA.

\subsubsection{The game of numbers between the helical antennas}

While going through the structural parameters of helical antennas, we noticed simple relationships between the structures, such as the radius of B-DNA (10 $)$ is equal to the sum of the pitch $(5.4 \AA)$ and the diameter (4.6 $\AA$ ) of $\alpha$-helix. Furthermore, the sum of B-DNA's pitch (34 $\AA$ ) and diameter $(20 \AA)$ is ten times the pitch $(5.4 \AA)$ of $\alpha$-helix.

As our interest in numbers grew, we began to look into the relationships between the antenna parameters. Considering two numbers equal when the percentage difference is less than $1 \%$, we observe a relationship between two antenna parameters in ratios involving integers and $\pi$ (Table 2). The percentage difference between the two numbers was calculated using the following formula: 


$$
\text { Percentage difference }=\frac{\text { value }_{\text {max }}-\text { value }_{\text {min }}}{\left(\text { value }_{\max }+\text { value }_{\text {min }}\right) / 2}
$$

Notably, we took the antenna parameters down to five decimal places in the angstrom unit for the percentage difference calculations.

These ratios are significant in the context of dipole antennas. A half-wave dipole antenna has a physical length to wavelength ratio of 1:2. Furthermore, for a half-wave dipole antenna, the effective length to physical length ratio is $2: \pi$, and the ratio between the effective length to wavelength is $1: \pi$. The effective length measures antenna efficiency in transmitting and receiving waves. For example, suppose the antenna's length equals the effective length of a wavelength. In that case, it means that the antenna transmits or receives that particular wavelength with the least power loss and that the antenna is highly efficient for the wavelength.

From various perspectives, many interpretations and explanations of a single ratio or combination of multiple ratios are possible. As helical antennas represent loop-dipole antennas, we will explain the ratios from the half-wave dipole antenna and loop antenna perspective to show that living systems have efficient wireless communication networks.

\subsubsection{Half-wave dipole antenna}

a) $96.83 \AA$ : $15.43 \AA \approx 2 \pi: 1 ; 193.37 \AA: 96.83 \AA \approx 2: 1$

One of the interpretations is that when the wavelength is $96.83 \AA$, as in the normal mode of $\alpha$-helix, the effective length of the antenna is equal to the wirelength of two turns of $\alpha$-helix ( $15.43 \AA$ x $2=30.86 \AA$ ). This means that two turns of $\alpha$-helix act as the ideal receiver of $\alpha$-helix wavelength $96.83 \AA$. Furthermore, because the wavelength $193.37 \AA$ is twice that of $96.83 \AA, 193.37 \AA$ also communicate efficiently with the wirelength of four turns of $\alpha$-helix (61.71 $\AA$ ).

Another interpretation is that when the physical length of an antenna is $96.83 \AA$ (wirelength of small BDNA), the wavelength is $193.37 \AA$ (normal mode wavelength of $\alpha$-helix). This means that $\alpha$-helix has the communication ability with a single turn of small B-DNA. Now, with a physical length of $96.83 \AA$, the effective length is $61.64 \AA$, which is equal to the wirelength of four turns of $\alpha$-helix $(15.43 \AA$ x $4 \approx$ $61.64 \AA$ ). Furthermore, when $15.43 \AA$ is the effective length, the physical length is $24.21 \AA$, and many antenna parameters equal multiples of the physical length $24.21 \AA$. Such as $24.21 \AA$ x $3 \approx 71.44 \AA$ (the wirelength of B-DNA); $24.21 \AA \mathrm{x} 4 \approx 96.83 \AA$ (wirelength of small B-DNA and normal mode wavelength of $\alpha$-helix); $24.21 \AA$ x $5 \approx 122.64 \AA$ (self-resonant wavelength of B-DNA); $24.21 \AA$ x $7 \approx$ $169.73 \AA$ (11 times the wirelength of $\alpha$-helix). These interlinked parameters indicate a network of communication among the antenna parameters. Furthermore, it appears that we can consider the wirelength of single turn small B-DNA (96.83 A) as a series of four segments with effective length 
$15.43 \AA$ and wirelength of single turn B-DNA (71.44 $\AA$ ) as a series of three segments with effective length $15.43 \AA$. Therefore, the wirelength of $\alpha$-helix (15.43 $\AA$ ) also acts as an ideal receiver for the wirelengths of B-DNA. As a result, if any wavelength communicates with $15.43 \AA$ efficiently, it also communicates efficiently with B-DNA. The normal mode wavelengths of $\alpha$-helix $96.83 \AA$ and 193.37 $\AA$, for example, communicate efficiently with $15.43 \AA$. Additionally, $15.43 \AA$ also acts as the physical length of a short dipole antenna for the wavelength $154.69 \AA$ (normal mode wavelength of $\alpha$-helix), indicating the wavelength $154.69 \AA$ also can communicate with the segment.

b) $96.83 \AA$ : $19.34 \AA 2 \approx: 1$

Even the normal mode wavelength of a single turn of $\alpha$-helix (19.34 $\AA$ ) communicates with B-DNA efficiently. The wirelength of a single turn of small B-DNA (96.83 $\AA$ ) is equal to the five multiples of $19.34 \AA$, which is similar to harmonic antennas or long wire antennas where the wirelength represents the multiples of a wavelength. Harmonic antennas have the advantage of having higher gain and directivity than half-wave dipole antennas.

The effective length for wavelength $19.34 \AA$ is $6.16 \AA$, and the multiples of effective length match other antenna parameters. Such as 2 x $6.16 \AA \approx 1 / 10$ of the $122.64 \AA$ (self-resonant wavelength of B-DNA); $3 \times 6.16 \AA \approx 1 / 10$ of the $182.88 \AA$ (wavelength of B-DNA); 5 x $6.16 \AA \approx 2$ x $15.43 \AA$ (wirelength of $\alpha$ helix); 7 x $6.16 \AA \approx 10$ x $4.29 \AA$ (length of an amino acid of $\alpha$-helix); 9 x $6.16 \AA \approx 10$ x $5.51 \AA$ (length of an amino acid of small $\alpha$-helix); 11 x $6.16 \AA \approx 10$ x $6.8 \AA$ (length of a nucleotide of B-DNA); $15 \mathrm{x}$ $6.16 \AA \approx 10 \times 9.22 \AA$ (length of a nucleotide of small B-DNA). This indicates that $\alpha$-helix communicates efficiently with individual amino acids and nucleotides using the normal mode wavelength. Perhaps $\alpha$ helix also counts the individual amino acids and nucleotides using the normal mode wavelength.

c) $182.88 \AA: 58.06 \AA \approx \pi: 1 ; 58.06 \AA: 19.34 \AA \approx 3: 1 ; 58.06 \AA: 14.45 \AA \approx 4: 1$

The relationship, $182.88 \AA: 58.06 \AA \approx \pi$ : 1 , states that when the wavelength of B-DNA is $182.88 \AA$, the effective length of the antenna is $58.06 \AA$. Interestingly, the effective length (58.06 $⿱$ ) is equal to three times the normal mode wavelength of $\alpha$-helix (19.34 $\AA$ ) and four times the axial mode wavelength of $\alpha$-helix (14.45 $\AA$ ), which indicates that the effective length (58.06 $)$ operate as harmonic antennas. Furthermore, because even multiples of a wavelength have higher gain than odd multiples of a wavelength in a harmonic antenna, the axial mode of $\alpha$-helix perhaps communicates with B-DNA more efficiently in this condition than the normal mode wavelength.

d) $71.44 \AA: 19.85 \AA \approx 3.6: 1$; and a few more

While the wirelength, normal mode wavelength, and axial mode wavelength of $\alpha$-helix are all present as harmonic series in the B-DNA, the wirelength of small $\alpha$-helix (19.85 $\AA$ ) is present in the B-DNA in a different way. When we multiply $19.85 \AA$ with the number of amino acids per turn of $\alpha$-helix (3.6), we get the wire length of B-DNA (71.44 $\AA$ ). This means that $19.85 \AA$ A represents a single amino acid in 
B-DNA. As two turns of B-DNA contain $\approx 7$ segments of $19.85 \AA$ and 21 nucleotides (10.5 nucleotides/turn), each segment of $19.85 \AA$ represents three nucleotides. This could be how cells read the gene or codon sequence (3:1) in the genome. Interestingly, seven times of $19.85 \AA$ is almost equal to nine times of $15.43 \AA$, indicating the link between the wirelength of small $\alpha$-helix and wirelength of $\alpha$-helix.

When the wirelength of small B-DNA (96.83 $\AA$ ) is divided by 3.6, the value is $26.90 \AA$, which is approximately five times the pitch of $\alpha$-helix (5.4 $\AA$ ). Furthermore, considering the $\alpha$-helix pitch (5.4 $\AA$ ) as the radius, the circle's perimeter is almost equal to the B-DNA pitch (34 $\AA$ ). Moreover, the pitch of the B-DNA is five times the length of a nucleotide (6.8 $\mathrm{A})$. These interrelationships point towards another network of communication involving the pitch of $\alpha$-helix, the pitch of B-DNA, and the wirelengths of B-DNA.

\subsubsection{Loop antenna}

Interestingly, the perimeter or wavelength of a large loop antenna also represents a half-wave dipole antenna for which the effective length is the diameter of the loop. Therefore, we have also considered the wirelengths and the circumferences of the helices as loop antennas for the analysis. For the wavelength $14.45 \AA$ (circumference of $\alpha$-helix and axial mode wavelength), the effective length is 4.6 $\AA$, which is half the length of a nucleotide (9.22 $\AA$ ) of small B-DNA, indicating the mechanism by which $\alpha$-helix read the nucleotides of the small B-DNA. Furthermore, the multiples of $4.6 \AA$, similar to $6.16 \AA$, also match many antenna parameters. For example. 3 x $4.6 \AA=1 / 10$ of $138 \AA(\approx 7$ x $19.85 \AA$; $\approx 9 \times 15.43 \AA$ ); 6 x $4.6 \AA \approx 5 \times 5.51 \AA$ (length of an amino acid of small $\alpha$-helix). This indicates that the $\alpha$-helix also efficiently communicates with these antenna parameters using the axial mode. Additionally, for the wavelength $15.43 \AA$ (wirelength of $\alpha$-helix), the effective length is $4.91 \AA$, which is exactly the $1 / 25$ of the self-resonant wavelength of B-DNA (122.64 $\AA$ ). Moreover, for the wavelength $19.85 \AA$ (wirelength of small $\alpha$-helix), the effective length is $6.32 \AA$, which is exactly the $1 / 5$ of the selfresonant wavelength of $\alpha$-helix (31.69 $\AA$ ). As a result, it appears that multiple interconnected networks are operating simultaneously inside a cell.

We have demonstrated that the relationships of antenna parameters mean two antennas communicate efficiently. The interrelationship analysis reveals that the $\alpha$-helix antenna parameters are embedded in harmonic series within the B-DNA, indicating that the B-DNA and the $\alpha$-helix communicate efficiently through an interconnected network of antenna parameters. The relationships also signify the role of turns in biomacromolecular communication, with each turn of the helices functioning as a modular unit. (27) Literature suggests that biomacromolecular conformations and topology play a vital role in the cellular communication of living systems. (28-30) Therefore, further investigation using these numbers, conformations, and topology may yield valuable information about the cell signaling pathways, the role of the non-coding genome, and communication networks in living systems. 


\subsubsection{Simulation of B-DNA and $\alpha$-helix}

We simulated the helical antennas to understand the radiation characteristics. The single turn of B-DNA was designed in AutoCAD 2021, while the single turn of $\alpha$-helix was designed in ANSYS Electromagnetics Suite 18.1. On ANSYS HFSS, the following simulation settings were used: all structures had a radius of $0.2 \AA$, the material was 'perfect conductor,' the excitation was 'lumped port,' an 'air box' was created at $\lambda / 4$ distance from the surface of the structures, and the Perfectly Matched Layer (PML) was used as a radiation boundary for all wavelengths. The following was the setup for illustrating the far-field radiation pattern: Theta sweep was from $-180^{\circ}$ to $+180^{\circ}$, and Phi sweep was from $0^{\circ}$ to $360^{\circ}$ with a step size of one.

B-DNA emits an omnidirectional 3D radiation pattern at all wavelengths up to $122.64 \AA$. At and above 174.17 $\AA$, the 3D radiation patterns are directional, which justifies the higher gain in those wavelengths (Table 3). The highest gain is measured at $232.23 \AA(6.11 \mathrm{~dB})$, followed by $174.17 \AA(4.47 \mathrm{~dB})$ and $182.88 \AA$ ( $4.41 \mathrm{~dB})$ (Figure 2G-I). Among the lower wavelengths $(<174.17 \AA)$, the maximum gain $(3.63 \mathrm{~dB})$ is observed at the wirelength of B-DNA $(71.44 \AA$ ), which is also a cardioid at the E-plane (elevation plane) (Figure 2B-C). At the H-plane (azimuthal plane), B-DNA shows cardioid with wavelengths of $58.06 \AA$, $96.83 \AA$, and $232.23 \AA$ (Figure 2A, D, I). Furthermore, the radiation shows mutual coupling with $116.11 \AA$ and $122.64 \AA$ (Figure 2E-F).

$\alpha$-helix shows a unidirectional radiation pattern with wavelengths $14.45 \AA, 15.43 \AA, 232.05 \AA$ and $251.38 \AA$ (Figure 3A, D, J). The maximum gain is at $251.38(5.33 \mathrm{~dB})$, followed by $232.05 \AA$ (4.98 $\mathrm{dB}), 15.43 \AA$ (4.92 dB), and $14.45 \AA(4.55 \mathrm{~dB})$. The radiation pattern is cardioid in the E-plane and Hplane with $14.45 \AA, 15.43 \AA$, and $232.05 \AA$ (Figure 3B, C, E, F, K, L). In contrast, $\alpha$-helix shows cardioid only at the H-plane with $19.34 \AA$, $19.85 \AA, 31.98 \AA$, $77.35 \AA, 174.03 \AA$, and $193.37 \AA$ (Figure 3G, H, I). Interestingly, $\alpha$-helix shows omnidirectionality at all the wavelengths of B-DNA (excluding $232.23 \AA$ ).

The cardioid pattern represents wideband, unidirectional radiation with a high front-to-back ratio, which results in improved performance in a multipath environment. Furthermore, a loop-dipole radar system also uses a cardioid radiation pattern to determine direction. As a result, it is possible that biomacromolecular helical antennas, which are also loop-dipole systems, use cardioid radiations to determine the direction and control cellular activities in a crowded intracellular environment.

High-gain antennas provide more precise signal targeting and are critical for long-range wireless communication. Increasing the number of turns and arranging the helices in different geometries are two common ways helical antennas achieve higher gain. The average number of turns in $\alpha$-helix is three; longer helices with more turns are present in super secondary motifs such as coiled-coil structures. (31) As a result, these coiled-coiled structures perhaps have increased sensitivity and range extension due to their higher gain. Furthermore, the $\alpha$-helices arrange themselves in variously patterned designs 
such as spirals, sheets, and rings in proteins. For example, in the spike protein of SARS-CoV-2, $\alpha-$ helices are present in hexagonal and triangular patterns (Figure 1C-D). As a result, it appears that $\alpha$ helices replaced dipole rod antennas in living systems, resulting in a diverse range of antenna systems for various biological functions.

Tapering the ends of helical antennas is another way to increase their gain. (32) Therefore, it appears that $3_{10}$ helices, another common helical secondary structure of proteins present at the $\mathrm{N}$ and $\mathrm{C}$ termini of $\alpha$-helices and have a smaller diameter than $\alpha$-helix, create a smaller diameter tapering ends and increases gain. Interestingly, another helical secondary structure, the $\pi$-helix, is generally present in functionally important sites of proteins between the $\alpha$-helix chains and causes the $\alpha$-helical structure to have a non-uniform diameter. Indeed, helical antennas with non-uniform diameters increase antenna bandwidth, justifying the presence of $\pi$-helices in functional sites. (33) Therefore, from the perspective of antenna theory, the $\alpha$-helices also have the necessary modifications with improved properties for various biological functions.

\subsection{Role of $\beta$-Strand}

The $\beta$-strand, which has a zigzag-like protein backbone, is another crucial and abundant secondary structure of proteins. The pitch of a $\beta$-strand is usually $7 \AA$, and the angle between the zigzag arms is $109.5^{\circ}$ due to the tetrahedral chemical bonding of the $\mathrm{C} \alpha$ atom.

We assumed that the $\beta$-strand acts as a zigzag antenna, with each unit, also functioning as a Vee-antenna (Figure 1E). Interestingly, the antenna parameters of the $\beta$-strand show a striking resemblance to antenna parameters of $\alpha$-helix (Table 4). Notably, with an angle of $109.5^{\circ}$ and an aperture width of 7 $\AA$, the length of the $\beta$-strand sidearm is $4.29 \AA$, which is exactly equal to the length of each amino acid of $\alpha$-helix ( $15.43 \AA / 3.6=4.29 \AA$ ). Using $4.29 \AA$ as the arm length, the wavelength of the zigzag antenna is $1.57 \AA$ (SI Eq. 1.2), (34-36), and the wavelengths of the Vee-antenna are $0.87 \AA, 2.42 \AA, 4.70 \AA$, and $5.55 \AA$ (SI Eq. 1.3). (37) The zigzag antenna wavelength (1.57 $⿱$ ) relates to the wirelength of small $\alpha$ helix (1/100 of $19.85 \AA$ x 8 or $1 / 4$ of effective length $6.32 \AA$, of wavelength $19.85 \AA$ ). The Vee-antenna wavelengths also relate to B-DNA and $\alpha$-helix. For example, the wavelength $5.55 \AA$ is the length of each amino acid of the small $\alpha$-helix antenna (19.85 $\AA$ /3.6=5.51 $\AA$ ), while the wavelength $0.87 \AA$ is half of $1.74 \AA$, which is $1 / 100$ of $174 \AA$, a wavelength for normal mode operation of both B-DNA and $\alpha$-helix. Therefore, it is evident that the $\beta$-strand, which has the characteristics of two different types of antennas, can communicate with B-DNA and $\alpha$-helix.

However, as a single strand is not favorable energetically, two or multiple strands self-assemble to form complex structures. Interestingly, if we replace $\beta$-strands with the dipole rod antennas, all the selfassembled structures of $\beta$-strands also resemble different types of existing antennas and antenna arrays. The antiparallel $\beta$-pleated sheet, for example, resembles a sectoral horn antenna, twisting between consecutive strands resembles a bowtie antenna, and $\beta$-barrels look like barrel antennas. To further 
investigate, we calculated the wavelengths of the antiparallel $\beta$-pleated sheet structure as horn antenna using the aperture width and slant height as $7 \AA$ and $4.29 \AA$, respectively (SI Eq. 1.4). Surprisingly, the wavelengths relate to the helical antenna parameters. The E-field wavelength is $5.72 \AA$, which is nearly 1/4 of effective length $(11.37 \AA$ ) of wavelength $71.44 \AA$, and the H-field wavelength is $3.81 \AA$, which is almost $1 / 4$ of the wirelength of $\alpha$-helix $(15.43 \AA)$ or 4 times the wavelength of water $(0.95 \AA)$.

It is difficult to know exactly the dimensions of these self-assembled structures, as not only do the arm's length and angle vary, but the gap between the strands also varies with the oscillation of proteins. However, in the case of an antiparallel $\beta$-pleated sheet, if the interstrand gap is $3.5 \AA$, as observed in silk and $\beta$-keratin, the structure displays some interesting game of numbers (Figure 1F). In the case of the rectangle with sides of $3.5 \AA$ and $4.29 \AA$, the perimeter (15.57 $\mathrm{A}$ ) turns out to be the wavelength of $\beta$-strand as the zigzag antenna. Furthermore, the perimeter of the rectangle with sides of $3.5 \AA$ and $7 \AA$ is $21 \AA$, which is $1 / 3$ of the axial wavelength of B-DNA (62.83 $\AA$ ). Additionally, the perimeter of the triangle (with sides of $4.29 \AA$, $4.29 \AA$, and $7 \AA$ ) is $15.57 \AA$, which is exactly same as the side 1 . These characteristics are comparable to those of loop antennas, in which the antenna's resonant wavelength is the perimeter of the loop. Curiously, the perimeters of the equiareal squares for side 1 and side 2 are $15.49 \AA$ and $19.80 \AA$, which also match the antenna parameters of $\alpha$-helix. Therefore, similar to $\beta$ strand, it appears that these assembled structures of $\beta$-strands also represent various types of antenna integrated complex communication systems.

Moreover, it seems that the aperture of a single unit of an antiparallel $\beta$-pleated sheet represents a twosegment aperture, with each segment having a width of $3.5 \AA$. Such segmented aperture antennas are usually for multiple beam formations to track the target. Notably, the zigzag and Vee antennas also have the beam-forming ability, and antiparallel $\beta$-pleated sheets are present in those proteins, namely receptors and enzymes that require the precise direction-finding ability to operate.

Based on the preceding discussions, it is clear that all the biomacromolecular antennas ( $\beta$-strand, $\alpha$ helix, and B-DNA) formed complex geometries to communicate efficiently with one another. Furthermore, it appears that wireless communication is required for any biomolecular activity. This is why losing the unique geometry results in losing molecular functions. Understanding how different geometries of biomacromolecular antennas operate and coordinate could reveal vital insights into the communication networks of living systems.

\subsection{Transmission lines and other structures of living systems}

\subsubsection{Role of cytoskeletal systems}

Because transmission power in a wireless communication system decreases proportionally to the square of the distance, transmission lines are preferable to wireless communication for long-distance communication. There are three types of transmission lines: a) open wireline, b) coaxial cable line, and 
c) waveguide. The open wire system is the simplest type of transmission line, and it can operate in Traverse Electromagnetic (TEM) mode. Coaxial cables, which are widely used to transmit high voltage and high-frequency signals, have less crosstalk than open wirelines and operate in TEM mode. Waveguides have a higher power handling capacity and less attenuation at higher frequencies than coaxial cable, but they only support Transverse Electric (TE) or Transverse Magnetic (TM) mode. In this section, we will conceptually compare the characteristics of cytoskeletons with those of transmission lines. (38-40)

\subsubsection{Microfilaments}

A Microfilament (MF) is a twisted pair of two F-actin polymer strands. F-actin polymers are composed of G-proteins, and each G-protein has multiple $\alpha$-helices and $\beta$-strands spreading over the protein's various planes (Figure 4A). We have already demonstrated that $\alpha$-helix and $\beta$-strands operate as antennas; therefore, microfilaments appear to work as twisted pair transmission lines with inbuilt antennas. The integrated antennas receive signals coming from various directions, and the twisted pair transmits them in both directions simultaneously. Furthermore, the twisted F-actin pair adopt a lefthanded supercoil twisting, possibly to cancel out the interference from the predominant right-handed circularly polarized waves. Interestingly, the diameter of microfilaments (70 $)$ is nearly identical, and their pitch (720 $\AA$ ) of the twists is almost ten times that of the wire length of a single turn of B-DNA (71.44 $\AA$ ), which indicate that the twists have resulted in another layer of superimposed helical antennas that can communicate with B-DNA and $\alpha$-helix.

\subsubsection{Extended coiled-coil structures}

Extended coiled-coil structures, such as those found in tropomyosin and myosin chains, seem to operate similarly to a twisted pair/multiwire collinear helical array antenna system, combining the benefits of twisted pair/multiwire transmission lines, collinear array antennas, and helical antennas (Figure 4B). (41) In a collinear array antenna, two or more similar antennas, often dipole antennas, are stacked vertically to enhance directivity, minimize minor lobes, increase the signal-to-noise ratio, and reduce power wastage. It appears that living systems substituted the dipole rod antennas with the helical antennas in the transmission line for better directivity, higher operating frequency, and wider bandwidth (axial mode of operation). Additionally, because the helical antennas are omnidirectional in normal mode, they remain linked to nearby structures. As a result, the extended coiled-coil design serves as an efficient transmission line required for various biological functions. Interestingly, the pitch of the coiled-coil structure ( $140 \AA$ to $150 \AA$ ) is approximately twice the wire length of a single turn of B-DNA, indicating that these superimposed helical coils play an additional role in the transmission line. Like microfilaments, the twists in the superimposed coils are primarily left-handed, possibly to reduce interference with right-handed circular polarised waves. 
In physiological conditions, the main chain of proteins perhaps carries a signal unidirectionally from $\mathrm{N}$-terminal (amine as electron donor group) to C-terminal (carboxyl as electron withdrawal group) because of the field gradient. (42) Although more study is required to understand the directional conductivity and electronic behaviors of proteins and amino acids, this directional conductivity signifies the differential distribution of $\mathrm{N}$ and $\mathrm{C}$ terminals of proteins across organelles and cell membranes. (43) The directional conductivity along the protein chains also highlights the parallel and antiparallel arrangement of chains in $\beta$-sheets, $\alpha$-helices in the coiled-coil structures, and other transmission lines of a cell.

\subsubsection{Intermediate filaments}

Intermediate filaments (IF) are primarily composed of different proteins in various cells (Figure 4C). $(44,45)$ These proteins create coiled-coil dimers by winding two parallel helical structures, and two of these coiled-coil dimers assemble in opposing orientations to form a protofilament. Then about eight protofilaments twist together like a rope to form an intermediate filament. This opposing orientation of dimers is interesting. This is similar to a coaxial transmission line, in which the magnetic fields of two oppositely directed current-carrying conductors would cancel each other out, resulting in less external interference. As a result, intermediate filaments provide the combined advantage of coaxial transmission lines, twisted-pair collinear helical antenna array-based transmission lines, and multiwire coiling. Furthermore, the length of a single $\alpha$-helical rod domain (350 A) indicates that the high gain helical antennas form the core of the intermediate filaments.

\subsubsection{Microtubules}

Microtubules (MT) are hollow cylindrical structures mainly composed of alpha and beta-tubulin subunits (Figure 4D). The hollow cylindrical structure indicates that microtubules possibly operate as flexible circular waveguides. The flexible circular waveguides are easily twistable and allow mechanical movement, giving a cell an added advantage. Furthermore, the circular waveguide's first dominant mode is TE11, which provides low attenuation per unit length, and the second dominant mode is TM01, which is rotationally symmetrical. The inner diameter of microtubules ranges from $110 \AA$ to $150 \AA$. Considering the inner diameter of microtubules as $150 \AA$, the cut-off wavelengths of TE11 and TM01 are $\approx 256 \AA$ and $\approx 196 \AA$, respectively (SI Eq. 1.5). It is the primary reason we considered the wavelengths of the B-DNA and $\alpha$-helix up to $256 \AA$. Furthermore, with an inner diameter of $110 \AA$, the cut-off wavelengths of TE11 and TM01 are $\approx 188 \AA$ and $\approx 144 \AA$, respectively, which justifies transmitting all the wavelengths of a single turn of helical antennas through the microtubular waveguide system.

Microtubule-organizing centers such as the basal bodies, centrosomes, and spindle pole bodies, besides serving as microtubule nucleation sites, possibly also serve as special waveguide components such as couplers, dividers, and multiplexers. (46) The basal body of the ciliary-flagellar system, for example, 
resembles a rotating joint coupler between the intracellular microtubular waveguide and the extracellular ciliary-flagellar waveguide system. This allows the extracellular and intracellular waveguides to move independently, resulting in stable back-and-forth communication between the internal and external environments. Furthermore, tubulin proteins, via their $\alpha$-helices and $\beta$-strands, perhaps act as couplers between the intra-waveguide propagating and extra-waveguide signals. $(47,48)$ This also explains how cilia and flagella-bearing cells and microorganisms sense their surroundings and transmit signals within. Moreover, it appears that the microtubules are the most critical transmission lines for communication. Therefore, any damage or loss of activity leads to cell death or apoptosis.

Although the cytoskeletons, as advanced transmission lines, justify many biological facts within a cell, a more in-depth investigation on how these transmission lines of the living systems operate is essential to understand the physiology of cells.

\subsubsection{Role of Histones and spermidines}

Histones are a family of basic proteins that helps in the regulation of transcription and compaction of DNA. Two molecules of four histones (H2A, H2B, H3, and H4) create an octamer complex around which 1.65 turns of DNA form a nucleosome via superhelical twists. (49) The octamer complex is dielectric because it has positively charged regions on the outside and negatively charged regions in the core. (50) Additionally, each histone has three $\alpha$-helices within its structured core that provide a magnetic field source resulting in a complex magnetodielectric environment within the octamer complex. Significantly, the 1.65-turn DNA resembles a metasolenoid structure composed of two connected single split-loop resonators that generate an extra magnetic field. (51) Moreover, the octamer complex, with its air gaps similar to a powdered core, may help reduce losses and give high inductance stability for the wrapped DNA. As a result, the DNA-wrapped octamer complex most likely functions as a complex and highly efficient loop-stick antenna with a magnetodielectric core capable of operating at very high frequencies without experiencing significant leakage effects. Because a loop-stick antenna primarily serves as a receiving antenna, the DNA must unwind from the histones in order to be active, transmit signals, and initiate transcription. This explains how histones control DNA transcription.

Interestingly, the toroidal DNA-protamine complex replaces the solenoidal DNA-histone complex in the mature sperms of a few species, including Homo sapiens. Indeed, the toroidal shape has benefits over the solenoidal shape in the compact environment found inside sperm heads. A toroidal shape generates a confined magnetic field, is less susceptible to interference, has wider bandwidth, and is a better candidate than a solenoidal structure inside any compact environment. This justifies why spermidine replaced histones for the compaction of DNA inside the sperm heads.

\subsection{Role of water molecules and chemical bonds}

\subsubsection{Role of water molecules and hydrogen bonds}


Water, another biomolecule, is essential for the survival of living systems. Although water is vital for molecular interactions, we still know little about how water works in biomolecule dynamics. $(52,53)$ To understand the role of a water molecule in molecular communication, we explored the properties of water molecules as Vee-antennas (Figure 5A). The similarity between the dipole character of the O-H bond and dipole arms of a Vee-antenna supports our assumption. Using the $\mathrm{H}-\mathrm{O}-\mathrm{H}$ bond angle of $104.45^{\circ}$ and $\operatorname{arm}(\mathrm{O}-\mathrm{H})$ length of $0.96 \AA$, the wavelengths of a water molecule are $0.1984 \AA, 0.95 \AA$, $1.19 \AA$, and $0.55 \AA$ (SI Eq. 1.3).

We compared the antenna parameters of a water molecule with the antenna parameters of B-DNA, $\alpha$ helix, and $\beta$-strand to determine whether a water molecule also communicates with biomacromolecules. Surprisingly, even the antenna parameters of water molecules relate to biomacromolecules' antenna parameters (Table 5). For example, the arm length of a water molecule is $0.96 \AA$, which is almost $1 / 100$ of the wire length of a small B-DNA antenna (96.83 $\AA$ ). Intriguingly, a wavelength of a water molecule is $0.1984 \AA$, which is exactly $1 / 100$ of the wire length of the small $\alpha$-helix (19.85 $\AA$ ). Furthermore, the wavelength $1.19 \AA$ also relates to $19.85 \AA$ ( $1 / 100$ of $19.85 \AA$ x 6), and another wavelength $0.55 \AA$ is exactly equal to $1 / 10$ of the wavelength of the $\beta$-strand (5.55 $\AA$ ). Even summation of the two arms of the Vee antenna (1.92 $\AA$ ), another possible wavelength of a water molecule, matches with $\alpha$-helix's normal mode wavelength ( $1 / 10$ of $19.34 \AA$ ). These numerical correlations show that the water molecules operate as the short dipole and Hertzian dipole antennas to the biomacromolecular antennas and efficiently communicate with B-DNA, $\alpha$-helix, and $\beta$-strand.

Water produces a maximum gain in the simulation study of all the discussed biomolecules (Table 3). Water molecule has the highest gain with $0.1984 \AA$ (12.61 dB), followed by $1.19 \AA(8.96 \mathrm{~dB}), 0.55 \AA$ $(8.75 \mathrm{~dB})$, and $0.95 \AA(6.87 \mathrm{~dB})$. The wavelengths $0.1984 \AA$ and $1.19 \AA$ generate cardioid in the $\mathrm{H}$ plane (Figure 6B, D, F H). Furthermore, water molecules exhibit an array radiation pattern with side lobes with all wavelengths, except $0.1984 \AA$, which displays an omnidirectional array pattern (Figure 6A, C, E, G, I, J, K, L). (54) When excited by the wavelengths of B-DNA and $\alpha$-helix, it emits omnidirectional radiation in all the wavelengths except $\approx 232 \AA$ and $\approx 251 \AA$, which emit directional radiation. (55) Interestingly, water molecule produces cardioid with the common normal mode wavelengths of B-DNA and $\alpha$-helix. The cardioid is in both the E-plane and the H-plane for wavelength $\approx 232 \AA$, whereas the cardioid is only in the H-plane for wavelengths $\approx 58 \AA$ and $\approx 174 \AA$.

Omnidirectional antennas transmit and receive signals in all directions and are widely in use to continuously broadcast and receive signals for location, time, and navigation services. Because water molecules are present in all cells and living systems, it appears that the water molecules, acting as omnidirectional antennas, provide continuous broadcasting systems and three-dimensional coordinates to the molecules, cells, and living systems. Additionally, because water molecules are also present as bound forms with biomacromolecules and have a high gain and directivity, the bound waters perhaps 
act as multiple external antennas to the internal biomacromolecular antennas, assisting in navigating one biomolecule to move in a specific direction towards another. (56-59) The characteristics of the water molecules also support their importance in the functional dynamics of biomacromolecules.

After noticing that the $\mathrm{O}-\mathrm{H}$ bond of a water molecule operates as an antenna, we investigated whether the hydrogen bond, another $\mathrm{O}-\mathrm{H}$ bond, also acts as an antenna. (60) The ability of water molecules to form hydrogen bonds with other water molecules is one of their most important properties. Interestingly, the hydrogen bond length of water molecules $(1.84 \AA$ ) matches the B-DNA wavelength (1/100 of 182.88 $\AA$ ). Furthermore, because each water molecule forms two hydrogen bonds involving its hydrogen atoms, the water molecule, together with the hydrogen bonds, represents another Vee-antenna with a bond angle of $104.45^{\circ}$ and arm length of $2.8 \AA(0.96 \AA+1.84 \AA)$ (Figure 5B). The wavelengths of the new Vee-antenna surprisingly also match with the antenna parameters of biomacromolecules (Table 5). For example, one of the wavelengths, $0.58 \AA$, is $1 / 100$ of the normal mode wavelength (58.06 $⿱$ ) of B-DNA. Another wavelength, $2.78 \AA$, is almost equal to half the wavelength of the $\beta$-strand $(5.55 \AA)$. The wavelength $1.61 \AA$, which generates a higher gain $(8.48 \mathrm{~dB})$ than other wavelengths, relates uniquely to the macromolecular antenna parameters. It is exactly $1 / 2$ of ( $34 \AA / 10.5)$ and $1 / 10$ of (58.06 $\AA / 3.6$ ), which indicates that even a water molecule can communicate and count the segments of helical antennas. Therefore, it appears that the hydrogen bonds, operating as antennas, modulate the characteristics of other molecular antennas and play a significant role in biomolecule dynamics.

Curiously, water molecule geometry, similar to $\beta$-strand/ $\beta$-sheet, exhibits an interesting game of numbers. The perimeter of the equiareal square for the circle of radius $0.96 \AA$ is $6.8 \AA$, exactly the length of a nucleotide ( $6.8 \AA$ ). Furthermore, the perimeter of the equiareal square for the circle of radius $2.8 \AA$ is $19.85 \AA$, which is exactly the wirelength of small $\alpha$-helix (19.85 $\AA$ ). Although more study is necessary to understand the significance of the relationships, it is fascinating to explore nature's beautiful geometries from the communication perspective. Interestingly, water molecules with hydrogen bonds also resemble other dipole antenna systems such as rhombic antennas and arrays of rhombic antennas (Figure 5C). (61)

Hydrogen bonds play critical roles in biological structure and function and are fundamental for sustaining life. One of the vital functions of hydrogen bonds is to stabilize the two strands of DNA, which are connected by two hydrogen bonds between adenine and thymine and three hydrogen bonds between guanine and cytosine. Interestingly, the hydrogen bonds between the strands ( $1.92 \AA$ and 1.82 $\AA$ A) also relate to the antenna characteristics of the B-DNA and $\alpha$-helix (Table 5). As a result, the linear array of hydrogen bonds between the DNA strands appears to be a typical broadside and end side array antennas, which is crucial for the DNA's function. Furthermore, because the intermolecular hydrogen bonds in other biological macromolecules vary widely in the range of $2.4 \AA$ to $3.3 \AA$, this variation in 
lengths most likely gives hydrogen bonds enough flexibility to communicate with a diverse range of biomolecules.

Therefore, as an essential part of the communication network, water molecules and hydrogen bonds play a vital role in the structure and stability of biomacromolecules, serve as biological coordinate systems, and assist in navigation and transmitting information.

\subsubsection{Role of chemical bonds}

Taking the cue from the relationship between the $\mathrm{O}-\mathrm{H}$ bond length and the helical antenna parameters, we explored the relationship between the other chemical bond lengths and the parameters of helical antennas. Because the chemical bonds oscillate continuously and the bond lengths of the bioatoms, i.e., $\mathrm{C}, \mathrm{H}, \mathrm{N}, \mathrm{O}, \mathrm{S}$, vary widely, it is difficult to correlate the features considering the bond lengths of biomolecules. As a result, we considered the average bond lengths of the atoms. (62) Surprisingly, all the average bond lengths equate to the helical antenna parameters (Table 6). For example, the average bond length of C-C is $1.54 \AA$, which is exactly $1 / 10$ of the wirelength of $\alpha$-helix (15.43 $\AA$ ). Similarly, the average bond length of $\mathrm{C}-\mathrm{O}$ is $1.43 \AA$, which is $1 / 10$ of the axial mode wavelength of the $\alpha$-helix (14.45 $\AA$ ). Furthermore, the C-S bond length is $1.82 \AA$, which corresponds to the wavelength of B-DNA (1/100 of $182.88 \AA$ ), and the $\mathrm{C}-\mathrm{H}$ bond length is $1.09 \AA$, which relates to the wirelength of $\alpha$-helix (1/100 of $15.43 \AA \times$ 7). Moreover, the average bond length of $\mathrm{C}=\mathrm{O}$ is $1.23 \AA$, which represents the $1 / 100$ of the self-resonant wavelength of B-DNA (122.64 $\AA$ ) and wirelength of $\alpha$-helix multiplied by eight (15.43 $\AA$ x 8). Not only do the chemical bonds of carbon match the helical antenna parameters, but also do the chemical bonds of the other atoms. For example, the average bond length for the disulfide bonds is $2.04 \AA$, which closely matches to $1 / 100$ of (15.43 $\AA$ x 13) and self-resonant wavelength of ten turns of $\alpha$-helix (207.48 $\AA$ ). The N-H's average bond length, $1.01 \AA$, is $1 / 100$ of $14.45 \AA$ multiplied by seven (14.45 $\AA$ x 7). The relationships show that all the chemical bonds are either short dipoles or Hertzian dipoles to biomacromolecular helical antennas indicating efficient communication between them.

Interestingly, although the average bond length of P-O $(1.60 \AA)$ and $\mathrm{P}=\mathrm{O}(1.48 \AA)$ correspond to multiples of $19.85 \AA$ (19.85 $\AA$ x $8 \approx 160 \AA$ ) and $9.22 \AA(9.22 \AA$ x $16 \approx 148 \AA$ ), the average bond length of a phosphate molecule ( $1.54 \AA$ ) is exactly $1 / 10$ of the wirelength of $\alpha$-helix (15.43 $\AA$ ). As a result, the average bond length of a molecule also appears to be important in the communication network.

Following that, we examined the chemical structure of nucleosides to assess what different bonds and their lengths indicate at the molecular level. (63) Upon comparing the C-C bond lengths in the rings of nucleobases and deoxyribose, the single $\mathrm{C}-\mathrm{C}$ bond of all nucleobases appears to communicate with $14.45 \AA$ (Figure 5E, F, G, and H). In contrast, the four C-C bonds of deoxyribose communicate only with $15.43 \AA$ (Figure 5D). Furthermore, the average $\mathrm{C}=\mathrm{C}$ bond length in thymine and cytosine is 1.34 $\AA$. In contrast, average $\mathrm{C}=\mathrm{C}$ bond length in adenine and guanine (1.38 $\AA$ ) corresponds to wirelengths 
of $\alpha$-helix (1/100 of the 7 times $19.85 \AA$, and 1/100 of the 9 times $15.43 \AA$ ). This demonstrates that chemical bond oscillation provides flexibility to a chemical bond to match with multiple antenna parameters of biomolecular antennas.

Interestingly, the effective length $(6.16 \AA)$ of the normal mode wavelength $(19.34 \AA)$ and the effective length (4.6 $\AA$ ) of the axial mode wavelength (14.45 $\AA$ ) of $\alpha$-helix represent the series of four $1.54 \AA$ segments and the series of three $1.54 \AA$ segments, respectively. We demonstrated that the effective lengths relate to various antenna parameters, including wirelengths of helical antennas and amino acid and nucleotide lengths. As a result, it appears that the $\mathrm{C}-\mathrm{C}$ bond and phosphate molecule are also capable of efficient communication with these antenna parameters. Moreover, the bond length $1.54 \AA$ also relates with the pitch of helical antennas, wirelength of small $\alpha$-helix, and the axial mode wavelength of B-DNA, indicating its leadership role in the interrelationships or communication network of the living system.

The chemical bonds that deserve special attention are $\mathrm{C}-\mathrm{N}$ and $\mathrm{C}=\mathrm{N}$ bonds, as their lengths vary widely in the range of $1.27 \AA$ to $1.47 \AA$ in various biomolecules. After evaluating the nucleobases, it seems those bond lengths also cluster around the numbers (1.30 $\mathrm{A}, 1.34 \AA, 1.35 \AA, 1.38 \AA$, and $14.5 \AA$ ) associated with macromolecular antenna parameters. However, we feel that these chemical bonds require more study because nucleobases, the genome's regulators, contain multiple such bonds and the gaps between the numbers linked to antenna parameters are not significant.

The analysis of chemical bonds, water molecules, and hydrogen bonds reveals that the chemical bonds act as antennas and communicate with all of the biomolecular antennas, forming a new layer in the interconnected communication network. Because chemical bonds such as $\mathrm{C}-\mathrm{C}, \mathrm{C}=\mathrm{C}, \mathrm{C}-\mathrm{H}, \mathrm{C}-\mathrm{N}, \mathrm{C}-\mathrm{O}$, $\mathrm{C}=\mathrm{O}, \mathrm{N}-\mathrm{H}$, and $\mathrm{O}-\mathrm{H}$ are present in almost all biomolecules, including DNA, RNA, proteins, sugars, lipids, and small molecules, it appears that all biomolecules communicate with one another. Because all biomolecules communicate with one another, they can understand their relative location, find directions, and operate efficiently in a crowded environment. This also explains how enzymes, transcription factors, and other molecules in low concentrations find their targets inside cells. Furthermore, as similar antennas communicate efficiently between them, the nucleotides can efficiently track signalling pathways because their structure matches those of many biomolecules involved in signalling pathways, such as ATP, GTP, and phosphates.

There are similarities between the multi-layered cell membrane, cell wall, and the icosahedral patterned structure of the capsid membrane with resonant cavity structures discussed in the literature. $(64,65)$ As a result, the cell envelopes and capsids may be resonant cavities that form standing waves in the antenna field, increasing the efficiency of antennas. Furthermore, because chemical bonds and macromolecules function as antennas, the resonant cavities are multiple antennae integrated that sense and respond to the environment as needed. This also indicates how the cytoskeletons work as multiple antennae 
incorporated sophisticated transmission lines within a cell. Interestingly, most biomolecules comprise heteroatoms, resulting in chemical bonds of different lengths within the molecule, similar to those seen in multi-band communication devices for better positioning precision.

Therefore, analysis and correlated biological facts justify our assumptions that B-DNAs, $\alpha$-helices, $\beta$ strands, water molecules, and chemical bonds operate as antennas, enabling high-speed molecular communication and coordination in living systems. It seems that a chemical bond represents a dipolar magneto-electric conducting wire, and the time-harmonic oscillations of electrons cause these dipoles to vary with time, resulting in a time-varying radiative field. (66) As a result, a molecule or macromolecule appears to be an antenna with a periodically magneto-electric conducting wire or L-C loaded transmission line, which has the added benefit of being broadband. (67-69) Indeed, many studies have found that dipole interactions are significant in molecular recognition. (70)

We have shown that biomacromolecules, water molecules, and even chemical bonds have communication ability or communicate among themselves. However, the ability to communicate necessitates processors and circuitry to interpret and respond to signals. Although the location of processors and circuitries is unknown, the fact that a chemical bond can operate as an antenna and communicate suggests that atoms have processors and circuitries for understanding and responding to signals. We still do not understand how an atom, composed of several subatomic particles, interprets and reacts to the information it receives; but, if we define the ability to sense, comprehend and respond to signals as "consciousness," the results show that "consciousness" occurs at the atomic level. (71-73) Because consciousness requires direction-finding ability and memory, the findings indicate that atoms also have these characteristics. (74)

We have seen that chemical bonds operate like small dipole antennas or Hertzian dipole antennas to biomacromolecules. However, because chemical bonds formed first and then biomacromolecules, it appears that the biomacromolecules were designed with the antenna parameters of the chemical bonds in mind. Interestingly, although the lengths of carbon atom bonds with bioatoms vary across biomolecules, the bond length of the carbon atom to other elements on the periodic table stays constant. Furthermore, the other bioatoms also form bonds of varying lengths with one another. Perhaps this ability of carbon atoms and other bioatoms to form chemical bonds of varying lengths provided them with abundant opportunities and resources to scale up characteristics of consciousness for the efficient communication infrastructures in living systems. Notably, while designing biomacromolecular structures for living systems, the atoms also considered the antenna properties of water molecules, which supports the existing hypothesis that macromolecular life originated in water. (75)

It is difficult to say whether atoms had a priori knowledge of creating a living system. However, given the complex and sequentially upgraded communication infrastructures that have evolved in living systems, it seems that the evolution did not occur as randomly as previously postulated. (76) Instead, 
the consciousness-mediated adaptive evolution appears to be systematically experimental to achieve that goal, with features of innovation, invention, and creativity. (77) Perhaps these experiments gave rise to biodiversity on Earth; at the same time, natural selection, or the ability to sense, adapt, integrate, and communicate with one's surroundings, determined the outcome or success of the experiments. (78)

We believe the $1 \%$ difference between the numbers is acceptable because biomolecules are structurally dynamic, and the antennas they represent are broadband antennas. Furthermore, a half-wave dipole antenna's physical length may not be precisely half of the wavelength due to the end effect. In a few relationships, notably the average bond length of C-N, S-S, and wavelength $0.95 \AA$ of a water molecule, the mismatch percentage exceeds $1 \%$. This is also reasonable because other biomolecular antennas, such as A-DNA, Z-DNA, and $\pi$-helix, may accurately match these interlinked antenna parameters. Perhaps, network optimization with all the parameters will give more relevant and accurate information about the communication architecture and dimensions of the biomolecules.

Therefore, part 1 of our manuscript demonstrates that living systems have advanced wireless and wired communication infrastructures consistent with biological facts. However, antennas transmit and receive electromagnetic radiation, and the calculated wavelengths of the antennas fall in the X-Ray and deepUV spectrums. As a result, it is improbable that biomolecules communicate with one another via these highly energetic and potentially harmful electromagnetic radiations. Because compatibility of the communication infrastructures in living systems is critical for this work, the next part is devoted to resolving the issue.

\section{Communication infrastructures of the Universe}

We know that living systems can adapt, integrate, and communicate with one another and their surroundings by using different waves such as sound waves and electromagnetic radiation. This piqued our interest in the biological implications of the recently discovered gravitational waves, also known as ripples in the space-time fabric. We thought why space-time fabric, which exists everywhere in the Universe, could not exist within living systems. According to Balanis, "electric charges are required to excite the fields but are not needed to sustain them and may exist in their absence."(79). After noticing that the antenna field can exist without electromagnetic radiation, we explored the links between the antenna field and the gravitational field.

\subsection{Antenna aperture and general relativity}

We assumed the magnetic dipole of an astronomical object as a magnetic dipole antenna, and devised the following equation linking the reactive near field radius of dipole antennas and Schwarzschild radius of Einstein's general theory of relativity. 


$$
\lambda_{s}=(0.62)^{2} \frac{(D)^{3}}{(\operatorname{ReNFR})^{2}}
$$

Where, ' $\lambda_{s}$ ' represents the Schwarzschild radius of the astronomical object, 'D' represents the largest dimension of the antenna or the length of a magnetic dipole antenna, and 'ReNFR' denotes reactive near field radius of the antenna. Notably, equation 2.1 is also the equation to calculate the reactive near field radius of a dipole antenna where ' $\lambda_{s}$ ' is the operating wavelength of the dipole antenna.

To develop the equation, we assumed that the Schwarzschild radius, or event horizon radius, represents the radius of the circular effective aperture of a magnetic dipole antenna. When the aperture serves as receiving system, the field propagates inward at the speed of light towards the antenna system, implying that the escape velocity must be greater than the speed of light to escape from the aperture. This is also a characteristic of the event horizon. The analogy between the event horizon and the aperture justifies the Schwarzschild radius as the aperture radius. Therefore, the equation indicates that the Schwarzschild radius of the astronomical object is the operating wavelength of the magnetic dipole antenna, which determines the ReNFR of the astronomical object. (80) This also means the gain of the magnetic dipole antenna is a constant $\left(4 \pi^{2}\right)$ (SI Eq. 1.6).

To understand what equation 2.1 indicates for the solar system, we calculated the ReNFR of the Sun considering the Schwarzschild radius (2953 m) of the Sun as the operating wavelength and the diameter of the Sun $\left(1.391 \times 10^{9} \mathrm{~m}\right)$ as the length of the magnetic dipole antenna (Table 8). Notably, the Sun's ReNFR, or the outer boundary of Sun's reactive near field zone (ReNFZ), lies between Mars and Jupiter ( $\approx 4 \mathrm{AU}$ ), just outside the asteroid belt. Then, using the following formula, we calculated the radiating far-field boundary of the solar system to establish the solar system's antenna field zones.

$$
\text { Radiating far field zone (RaFEZ) } \geq \frac{2 D^{2}}{\lambda_{s}}
$$

The result reveals that the RaFFZ (Fraunhofer's zone) begins around 8759 AU, located in the Oort cloud region, more specifically in the inner Oort cloud region or Hills cloud region. We can deduce from the results that the zone between the ReNFR (3.95 AU) and the RaFFZ (8759 AU) is the solar system's radiating near field zone (RaNFZ) or Fresnel zone. Interestingly, the four planets inside the ReNFR (Mercury, Venus, Earth, and Mars) are smaller, non-volatile, and rocky. In contrast, the four planets inside the RaNFZ (Jupiter, Saturn, Uranus, and Neptune) are larger and gaseous. It appears that the rocky planets require the ReNFZ characteristics to form higher molecular weight elements. Furthermore, the planets of the RaNFZ also have ring systems implying that there could be a link between the RaNFZ's characteristics and the planets' ring development. 
Following that, we used the planets' polar diameter as the length of the magnetic dipole antenna and their Schwarzschild radius as the operating wavelength to calculate the ReNFR of individual planets (Table 7). Intriguingly, the four planets inside the ReNFR of the Sun accumulated mass in quantity so that these planets' ReNFR is approximately half the Sun's ReNFR. Furthermore, the Earth's ReNFR is nearly equal to the diameter of its orbit, and the Earth has the lowest ReNFR of any planet. Compared to the planets within the Sun's ReNFZ, interestingly, the planets within the RaNFZ have a ReNFR greater than or nearly equal to the Sun's ReNFR. The most surprising aspect of the data is the ReNFR of Saturn, which is almost 30\% higher than the ReNFR of the Sun. Importantly, Saturn has the most prominent ring system among the planets, implying a link between the ReNFR and the planets' ring systems.

Additionally, we modified equation 2.1 to include Newton's law of gravitation in the equation.

$$
\frac{c^{2} \lambda_{s}}{8 \pi^{2}}=(0.62)^{2} \frac{c^{2}}{8 \pi^{2}} \frac{(D)^{3}}{(\operatorname{ReNFR})^{2}}=\frac{G M}{4 \pi^{2}}
$$

Where 'c' represents the speed of light, 'M' is the mass of the astronomical object, and 'G' denotes the Gravitational Constant. According to Equation 2.3, the mass of an astronomical object is directly proportional to the cube of its dipole length and inversely proportional to the square of its ReNFR. This reveals that the mass is most likely the stored energy within the smallest sphere that can enclose the magnetic dipole antenna. $(81,82)$ Therefore, a longer magnetic dipole length means a larger volume of the sphere to store energy; thus, the mass will be greater; whereas, a longer ReNFR indicates higher radiating energy, implying a lower stored energy or mass. This is important as the equation suggests that the mass is located outside the event horizon, whereas we have traditionally considered the mass is present within the event horizon. Equation 2.3 also reveals that the astronomical object's density is inversely proportional to the square of the ReNFR.

We further modified equation 2.3 for the binary systems (central object and revolving object) to include Kepler's third law of planetary motion in the equation.

$$
\frac{R^{3}}{T^{2}}=\frac{c^{2} \lambda_{s}}{8 \pi^{2}}=(0.62)^{2} \frac{c^{2}}{8 \pi^{2}} \frac{(D)^{3}}{(\operatorname{ReNFR})^{2}}=\frac{G\left(M_{1}+M_{2}\right)}{4 \pi^{2}}
$$

Where ' $R$ ' represents the semi-major axis of the revolving object, ' $T$ ' represents the orbital period of the revolving object, and ' $\mathrm{M}_{1}$ ' and ' $\mathrm{M}_{2}$ ' are the masses of the central object and the revolving object, respectively. Notably, ' $\lambda_{s}$ ' is the aperture radius of the central object's magnetic dipole antenna. In comparison to equation 2.3, the sum of the masses in equation 2.4 is acceptable because the astronomical object, which revolves around another astronomical object, usually has a much lesser mass; thus, the influence of the revolving object's mass becomes negligible. 
To verify equation 2.4 and understand what Kepler's third law of planetary motion indicates, we calculated the operating wavelength of the solar system's planets (Table 7). The results show that four planets inside the ReNFZ operate around a single wavelength $(\approx 2953 \mathrm{~m})$ which is also the Schwarzschild radius of the Sun. In comparison, four planets of RaNFZ operate at slightly different wavelengths. Saturn has the longest operating wavelength $(\approx 2995 \mathrm{~m})$, followed by Uranus $(\approx 2962 \mathrm{~m})$, Jupiter $(\approx 2959 \mathrm{~m})$, and Neptune $(\approx 2950 \mathrm{~m})$ in decreasing order. The findings demonstrate that the connection between Kepler's third law of planetary motion and the Schwarzschild radius of the central object as the operating wavelength is justified and valid. Equation 2.4 also demonstrates that the Sun's operating wavelength or Schwarzschild radius influences the orbital distribution and orbital period of the solar planets. Notably, the operating wavelengths of Saturn, Uranus, and Jupiter are higher than the Schwarzschild radius of the Sun, with Saturn having the widest deviation. Considering that Saturn also has the most prominent rings, it is plausible that there are links between the formation of the ring systems and the wavelengths.

However, we now have a wavelength other than the Schwarzschild radius of the planet that influences the planet's ReNFR. Therefore, to understand the influence of the Sun's Schwarzschild radius as the operating wavelength, we calculated the ReNFR of the planets, considering the planet's polar diameter as the antenna length. The results show that the planets within the ReNFZ of the Sun have lower ReNFR than the planets in RaNFZ. Earth has the highest ReNFR among inner asteroid belt planets, followed by Venus, Mars, and Mercury in decreasing order. The ReNFR of planets within the RaNFZ falls further away from Sun; Jupiter has the highest ReNFR, followed by Saturn, Uranus, and Neptune. Notably, all of the planets' moons are present within the new ReNFR of the planets and are primarily rocky, as we previously observed. The result is significant because we now have two reactive near-field radii of a planet considering two different operating wavelengths. One ReNFR is when the planet is under the influence of the Sun, and another ReNFR is when the planet is operating independently. Notably, the gaseous planets without the influence of the Sun achieve the ReNFR similar to Sun; further investigation of this result with different planetary systems may provide us valuable information on how astronomical objects evolved in the Universe.

\subsection{Antenna gain and general relativity}

After observing that many solar system features, such as the structural arrangement, can be explained using the equation, we started exploring the other implications of the equations. As the gain of a dipole antenna varies exponentially with the wavelength, we varied the wavelength in multiples of 10 (Table 8). Interestingly, if we consider the wavelength of the Sun as $29.53 \mathrm{~m}\left(\lambda_{\mathrm{s}} / 100\right)$ and $2.953 \mathrm{~m}\left(\lambda_{\mathrm{s}} / 1000\right)$, the ReNFR of the Sun represents the semi-major axis of Pluto $(\approx 39.5 \mathrm{AU})$ and Heliopause $(\approx 125 \mathrm{AU})$, respectively. Furthermore, if we consider the wavelength as $100 \lambda_{s}$, the corresponding ReNFR of Sun is 
nearly the semi-major axis of mercury $(\approx 0.39 \mathrm{AU})$. The results show that the planetary distribution of the solar system spans from $100 \lambda_{s}$ to $\lambda_{s} / 100$ of the Sun.

The observation suggests that the lower wavelength than the Schwarzschild radius or higher gain indicates towards the features of the radiative zone, whereas, higher wavelength than the Schwarzschild radius or lower gain points towards the characteristics of the reactive zone. From the perspective of an aperture of an antenna, this is also a characteristic of a parabolic antenna system where wavelength should be less than aperture to have a radiative field, and if the wavelength is more than the aperture, the field is restricted within the ReNFR. Therefore, the event horizon of the Schwarzschild metric is the effective aperture of a parabolic antenna, the curved space-time within the event horizon represents the curvature of the parabolic antenna, and the singularity is the vertex of the parabolic antenna. Furthermore, while the receiver antennas represent black holes, the transmitter antennas are white holes, and the transceiver antennas have the features of both black holes and white holes.

As transmission and reception functions are typically co-located in antennas, transceiver systems are supposed to be more prevalent in the Universe than either the receiver or the transmitter system alone. Therefore, it appears that polar emissions such as galactic jets, stellar jets, or emissions from pulsars, Saturn, and Jupiter are transmissions from the astronomical objects' apertures. (83) Furthermore, Perhaps, astronomical objects use the aperture transmissions to communicate with other astronomical objects. This could be the reason why Jupiter's polar decametric radio bursts correlate with the Io's (Jupiter's moon) orbital period. $(84,85)$ Additionally, as the decametric radio bursts are of higher wavelengths than the Schwarzschild radius of Jupiter (2.82 m), it supports our observation that emission with a wavelength greater than the $\lambda_{s}$ is needed to communicate with objects in the reactive field and emission with a wavelength less than the $\lambda_{s}$ is required to communicate with objects in the radiative field. Moreover, the modulation of the emissions observed in the Universe also indicates that the emissions are probably for communication purposes.

We have shown how the Schwarzschild metric's event horizon representing the effective aperture explains many features of the Universe and solar system. In addition to the event horizon, another exciting feature of the Schwarzschild metric is the photon sphere. (86) Interestingly, the photon sphere, whose radius is 1.5 times Schwarzschild's radius and represents the lower bound for any stable orbit of non-rotating black holes, also has an analogy in the antenna theory. If the aperture radius is 1.5 times the wavelength, the new aperture represents a constant field circular radius where the field pattern is symmetrical. (79) Therefore, the photon sphere represents the parabolic antenna system's constant field aperture or physical aperture. To understand the effect of the physical aperture of the Sun over the solar system, we calculated the ReNFR of the Sun using the operating wavelength as $1.5 \times \lambda_{\mathrm{s}}(\approx 4430 \mathrm{~m})$ (Table 8). The results show the ReNFR of the Sun is $\approx 3.23 \mathrm{AU}$, which represents exactly the end of the asteroid belt. Additionally, if we consider the wavelength as $\left(1.5 \times \lambda_{s}\right) / 100$, the ReNFR of the Sun 
becomes 32.30 AU, which represents the beginning of the Kuiper's belt. Moreover, considering ( $1.5 \mathrm{x}$ $\left.\lambda_{s}\right) / 1000$ as wavelength, the ReNFR becomes $102.15 \mathrm{AU}$, mid of heliosphere from where the solar wind speed in the heliosheath starts decreasing as detected by Voyager 1. (87) Importantly, considering 1.5 $\mathrm{x} \lambda_{\mathrm{s}}$ as wavelength, the far-field of the Sun begins at $\approx 5839 \mathrm{AU}$, at the start of the Inner Oort Cloud.

Therefore, it appears that the asteroid belt, Kuiper's belt, heliosphere, Oort cloud resulted from the aperture edge's diffraction effect (scattering), and this scattering from the edge of the antenna is the cause of the numerous small astronomical bodies and turbulence in those zones. Moreover, it also appears that the backward scattering formed the asteroid belt, whereas forward scattering formed the Kuiper's belt and Oort cloud. This indicates that the Sun most likely acts as a magneto-electric dipole antenna rather than acting only as a magnetic dipole antenna. Perhaps the magnetic dipole effect, which is proportionally greater than the electric dipole effect, produces unbalanced dipoles that cause the backscattering and the forward scattering. (88-90) The presence of both a magnetic dipole and an electric dipole in the Sun implies that it operates not only in TE mode but also in TM mode. Antennas that operate in both TE and TM modes simultaneously have higher bandwidth. (91) Importantly, spiral antennas that are geometrically comparable to spiral galaxies, the most prevalent type of galaxies, also operate in TE and TM modes.

Until now, we have mainly discussed the Schwarzschild metric of the Einstein field equation, which is for the stationary or non-revolving black holes. However, a revolving black hole follows Kerr's metric system, where the aperture or ergosphere is elliptical. Because astronomical objects are also revolving, the aperture for astronomical objects would be elliptical. The elliptic aperture has advantages over the circular aperture, mainly when the feed is not symmetric. The elliptic aperture produces a more concentrated beam at the focal point and indicates that the underlying magnetic dipole is also elliptical, giving it a wider bandwidth. Notably, because the minor diameter of an elliptical aperture determines the resonant wavelength of the dipole, the equations will not change, as the minor diameter is also the Schwarzschild radius of Kerr's metric system. As a result, the elliptical reflections or elliptical modal expansions from these elliptical antennas most likely caused the ellipse-shaped planetary orbits.

From the above findings and discussions, it is clear that the aperture of the antenna, as described in general relativity, together with the magnetic dipole antenna of astronomical objects, plays a vital role in determining the structural arrangements and emissions in the Universe. Furthermore, it also appeared that the solar system is a model example of an inductively coupled near field communication device with features of capacitive and scattering coupling.

\subsection{Transmission lines of the Universe and Antenna optimization}

We will conceptually explain other facts of the Universe and astronomical objects from the perspective of antenna theory in this section. 
According to antenna theory, magnetic dipole antennas primarily operate in the TE mode. The TE mode has the advantage of providing space with a higher impedance than the TM mode, which improves antenna performance, isolates antenna feeds from each other, and allows antennas to be located close together in a limited area. However, the TE mode requires a waveguide structure to propagate. As the strength of the magnetic field increases gradually towards the poles before decreasing again, the entire set will likely operate as a graded-index magnetic waveguide. $(92,93)$ Therefore, as the waveguides of the planets appear to merge into a larger waveguide of a star, the waveguides of the stars would most likely integrate with the waveguide of a supermassive object at the galaxy's center. Following that, waveguides of supermassive objects would connect with a central waveguide of galaxies, forming the Universe's transmission line. As a result, it appears that the magnetic highways, filaments, laniakea, cosmic webs, and recently reported tunnel-like magnetic structures are all part of the same transmission line. (94) (95) One of the advantages of the space waveguide system is that it provides a channel for an offset feed system to the apertures or parabolic antennas of astronomical objects, which reduces crosspolarization and increases the antenna efficiency further.

The solar system waveguide appears to be an elliptically tapered horn antenna due to its field zones, with the apex of the horn at the Sun and the aperture ending at the beginning of RaFFZ. Interestingly, the flaring of the horn antenna follows an exponential curve for the solar system as the distance between different regions in the solar system varies as multiples of 10 (Mercury $\approx 0.39 \mathrm{AU}$; ReNFR $\approx 3.95 \mathrm{AU}$; Pluto $\approx 39.5 \mathrm{AU}$ ). The advantage of exponential flaring is that it provides progressive impedance matching. However, the horn also has few transitions with sudden flarings, resulting in a hybrid constant directivity horn. One such transition occurs at the junction of ReNFZ and RaNFZ. The sudden transition from ReNFZ to RaNFZ inside the horn antenna resulted in the reflections that helped form the asteroid belt. As a result, it appears that the formation of the asteroid belt can be described in a variety of ways, and multiple interconnected mechanisms are at work in the formation of the scattering zones.

We observed that the planets within the ReNFZ operate in a single wavelength, whereas the planets within the RaNFZ operate in multiple wavelengths. It indicates that the ReNFZ operates like a singlemode system, whereas that RaNFZ operates like a multimode system. Therefore, within the multimodal waveguide, the dispersion of the operating wavelengths most likely formed the planetary ring systems. (96) It seems that the planets and the moons, with their field distribution inside the solar system waveguide, have also created hybrid banded waveguide structures, different grating, and corrugated systems to reduce losses and optimize the waveguide. (97) It will be interesting to explore the space transmission lines to understand how the transmission system works in the Universe and to develop various technologies such as electromagnetics and acoustics by mimicking them.

As previously stated, the strength of the magnetic field in a magnetic dipole is zero at the dipole's magnetic equatorial plane and highest at the poles. Perhaps, this gradient of field distribution towards 
the equatorial plane can explain why the rotation of one astronomical object around another, including the orbiting of planets around the Sun, occurs in a single plane. Another reason supporting single plane distribution is the nodal plane of standing waves within the waveguide. Field scattering and the influence of other fields are likely the reasons behind any deviation from the single plane. For example, the higher orbital inclinations of a few objects in the asteroid belt and Kuiper's belt perhaps because of the scattered fields in those locations.

We discussed that the orbits of the astronomical objects represent the mode-expanded regions within the flared waveguide. Literature also suggested that magnetic fields play a significant role in the stars and planets forming process. (98) The magnetic dipoles found inside astronomical objects are most likely induced magnetic dipoles formed by loop, helical and spherical antenna systems of fields. According to the antenna literature, folded spherical helix antenna systems are among the most efficient antenna systems for creating a magnetic dipole inside them. $(99,100)$ Therefore, it is plausible that the astronomical objects also have characteristics of folded spherical helix antennas and other forms of antennas that created the induced magnetic dipole inside them.

As previously discussed, nature has the mechanisms to optimize the transmission line. Thus, nature also should have mechanisms to improve the performance of astronomical antennas. (101) One way to enhance antenna efficiency is to ensure uniform current distribution. A shunt capacitor plate above the magnetic dipole is one of the possible ways that provide uniform current distribution. Thus, polar vortices or the low-pressure zone with overhead clouds at the planet's poles are most likely acting as a vacuum gap capacitor to achieve uniform current distribution. It is also delightful to observe that nature has also established ways to lower the temperature at the poles for minimizing thermal gradients and stabilizing the aperture that would have a deteriorating effect on the antenna's performance. Additionally, it seems that many astronomical objects contain dielectric and magnetic materials such as silicon dioxide and iron into the antenna structure to improve radiation efficiency. (102) Therefore, it is apparent that nature utilizes multiple interconnected mechanisms to improve the antenna structures and feed systems that increase the efficiency of the communication infrastructures of the Universe.

Throughout our discussions, we emphasized various astronomical facts concerning a single magnetic dipole antenna and its apertures. On the other hand, an astronomical object may have multiple magnetic dipoles or even multiple electrical dipoles in various configurations to shape the beam. (103) Additionally, the aperture does not have to consist only of parabolic antennas as stated previously but can include other types of antennas, such as slot antennas and lens antennas. Moreover, unlike in a monostatic system, the apertures do not have to be located only at poles to perform receiving and transmitting functions. A multistatic antenna system has two or more apertures in different locations for separate receiving and transmitting functions. The Sun, for example, operates as a multistatic system, with its sunspots acting as slot antennas. Interestingly, similar to slot antenna, the magnetic field 
strength is higher at the sunspots than anywhere else on the Sun. Besides, we have assumed the magnetic dipole antennas as the constant gain antennas, which may not hold everywhere. Furthermore, astronomical antennas may operate in various modes with different field patterns, both individually and in various geometrical shapes of groups, clusters, and arrays. The solar system, for example, is likely to function similarly to an inductively coupled planar strip spherical array antenna system or a planar Fresnel zone antenna system.

Therefore, innumerable possibilities are there on how these astronomical antennas operate and how nature optimized the characteristics of antennas and antenna fields in building up the most advanced, multi-antenna integrated communication infrastructures in the Universe. Numerous studies on general relativity, electrical engineering, optics, and metamaterials are underway. (104-107) These works most likely depict the actual properties of astronomical objects and the Universe. Furthermore, because antennas increase and regulate emissions, as documented in the literature, more study on coupling and resonances between astronomical antennas may provide plausible explanations for various emissions from astronomical objects. $(69,108-110)$ Understanding how these astronomical antennas and transmission lines work will enhance our knowledge regarding the operation of the Universe.

In part 2 of our manuscript, we have interpreted various aspects of Einstein's general relativity from the antenna theory perspective and linked it with Kepler's law of planetary motion and Newton's law of gravitation. We have also elaborated on many facts of the Universe, most notably the solar system, from the perspective of antenna theory. It is evident from the results that the gravitational field or the spacetime fabric is the field of the astronomical antennas, and the gravitational waves are the antenna field waves. As a result, it appears that the antenna field is the fundamental force of nature. Furthermore, because gravitational waves differ from electromagnetic radiation, the antenna field waves that nature's antennas use to communicate also exist without electromagnetic radiation.

The above findings indicate that the molecular antennas, macromolecular antennas, and living systems communicate, sense, coordinate and control their activities using antenna field waves or gravitational waves, just like astronomical antennas. This explains why wireless communication is compatible with molecules, cells, and living systems.

\section{Limitations and implications of the study}

While the results are exciting, the study has limitations and boundaries pertinent for discussion.

One significant limitation is our conclusion that consciousness or the complex circuitries of the antennas exist at the atomic level. We are still not sure where the consciousness exists; is it at the level of atoms as we have suggested or at the level of subatomic particles, or even down to the level of energy. However, if the communication perspective holds, nature has a purpose and everything of nature, from the atoms to the Universe, is conscious. (111) 
In the equations that link the gravitational field with the antenna field, the $4 \pi^{2}$ has its importance because $4 \pi^{2}$ is the constant gain of astronomical antennas. It also appears in the denominators of the equations involving the gravitational constant and the Schwarzschild radius. Considering the significance of $4 \pi^{2}$ and the exponential relationship of wavelength with field distribution, the constant 0.62 of the ReNFR equation perhaps would be 0.628 or $2 \pi / 10$. In that case, the more accurate modified equation will be

$$
\frac{R^{3}}{T^{2}}=\frac{c^{2} \lambda_{s}}{8 \pi^{2}}=\frac{c^{2}}{200} \frac{(D)^{3}}{(\text { ReNFR })^{2}}=\frac{G\left(M_{1}+M_{2}\right)}{4 \pi^{2}}
$$

It will change the calculated ReNFR slightly, but it will not affect the study's observations, discussion, and findings.

We observed that the dipoles and the resulting radiating fields are the common characteristics for all the structures, from chemical bonds to astronomical objects. Even water molecules, nucleic acids, amino acids, and secondary structures of proteins such as $\beta$-strands and $\alpha$-helices all form dipoles. (112) Although we described the dipoles classically, the principle of quantum antennas may better explain the structures' antenna properties. (113) Many studies have already reported the quantum phenomena in biological processes such as photosynthesis reaction and direction-finding by migratory birds. (114) Further investigation of many other aspects of communication infrastructures from the quantum mechanics perspective would provide invaluable insight on the subject. Notably, because dipoles also exist in the subatomic particles, the study's findings suggest that antenna field, antenna field zones, and field waves exist at the subatomic level as well.

Another observation concerns the ratios of antenna parameters in living systems. Surprisingly, the ratios resemble the music interval ratios. The presence of harmonic series, standing waves, and a relationship with seven indicate that music mathematics is vital in coordinating processes within a cell. Moreover, multiples of 10 or the common logarithm and antenna gain appear important in the relationship of chemical bonds with macromolecular antennas, similar to the solar system. Perhaps, this is associated with logarithmic perception at the molecular level and field zone interaction during contact mode communication. (115) Therefore, more studies from various perspectives are required to understand what is happening at a living system's atomic and molecular levels.

Although more research is needed to understand the communication infrastructures of living systems and the Universe, the findings have implications in many scientific fields. It will be interesting to investigate the role of antenna field, antenna field waves, and antenna field zones at the molecular scale to understand molecules' various physicochemical properties and interactions. Perhaps that will also explain how enzymes, molecules' self-assembly, and hydrophobic interactions function within a cell. 
Furthermore, applying knowledge from the astronomical scale to the atomic scale and vice versa will enhance our understanding at both levels.

There are numerous possibilities that the translation of this knowledge will allow us to explore new scientific arenas and develop technologies based on them. However, the study also warns that existing communication technologies may be interfering with nature's communication at all levels of the Earth, from living systems to the poles of the Earth, an issue that we must investigate and address thoughtfully. (116-118)

\section{Conclusion}

The study provides answers to many puzzles of biology and astronomy from the antenna theory perspective. The study signifies the molecular geometry in living systems, explains water's function, hints at how evolution occurred, and demonstrates how living systems perform high-speed coordinated actions and search out molecules in the crowded cell environment. In astronomy, the study links antenna field and gravitational field, which shows the antenna field can sustain the field waves separate from electromagnetic radiation, defines 'mass,' and explains the facts of the Universe, particularly the solar system.

Overall, the study questions the existence of 'randomness' in nature and suggests that the most efficient communication infrastructures exist within living systems and the Universe using the dipoles, the antenna fields, antenna field zones, and antenna field waves. We hope the study will help us understand our mother nature better than before and will aid in the development of new technologies for more harmonious and happier living.

\section{Acknowledgments}

We would like to thank our family, friends, and institute for supporting us.

\section{Contributions}

The assumptions, concepts, equations linking antenna field with gravitational fields, analysis, and interpretations are by SSR. Simulations, supplementary file, and figures are by BM. Calculations and tables are by both BM and SSR. Manuscript drafting is by SSR with the contributions of BM. The study is teamwork under the guidance of SSR. BM shares equal authorship with SSR. 


\section{References}

1. Scholkmann F, Fels D, Cifra M. Non-chemical and non-contact cell-to-cell communication: A short review. American Journal of Translational Research2013.

2. Mondal K, Chaudhury S. Effect of DNA Conformation on the Protein Search for Targets on DNA: A Theoretical Perspective. The Journal of Physical Chemistry B. 2020;124(17):3518-26.

3. Sheinman M, Bénichou O, Kafri Y, Voituriez R. Classes of fast and specific search mechanisms for proteins on DNA. Reports on Progress in Physics2012.

4. Foffi G, Pastore A, Piazza F, Temussi PA. Macromolecular crowding: Chemistry and physics meet biology (Ascona, Switzerland, 10-14 June 2012). Physical Biology2013.

5. Kučera O, Cifra M. Cell-to-cell signaling through light: Just a ghost of chance? Cell Communication and Signaling2013.

6. Farhadi A. Non-chemical distant cellular interactions as a potential confounder of cell biology experiments. Frontiers in Physiology. 2014;5(OCT).

7. Fels D. Electromagnetic cell communication and the barrier method. Fields of the Cell2015.

8. Halford SE, Marko JF. How do site-specific DNA-binding proteins find their targets? Nucleic Acids Research2004.

9. Rossi C, Foletti A, Magnani A, Lamponi S. New perspectives in cell communication: Bioelectromagnetic interactions. Seminars in Cancer Biology2011.

10. Veksler A, Kolomeisky AB. Speed-selectivity paradox in the protein search for targets on DNA: Is it real or not? Journal of Physical Chemistry B. 2013;117(42).

11. Thackston KA, Deheyn DD, Sievenpiper DF. Limitations on electromagnetic communication by vibrational resonances in biological systems. Physical Review E. 2020;101(6).

12. Amdursky N. Electron Transfer across Helical Peptides. ChemPlusChem2015.

13. Artés JM, López-Martínez M, Díez-Pérez I, Sanz F, Gorostiza P. Nanoscale charge transfer in redox proteins and DNA: Towards biomolecular electronics. Electrochimica Acta. 2014;140.

14. Brizhik LS, Luo J, Piette BMAG, Zakrzewski WJ. Long-range donor-acceptor electron transport mediated by $\alpha$ helices. Physical Review E. 2019;100(6).

15. Chen H, Fraser Stoddart J. From molecular to supramolecular electronics. Nature Reviews Materials2021.

16. Michaeli K, Beratan DN, Waldeck DH, Naaman R. Voltage-induced long-range coherent electron transfer through organic molecules. Proceedings of the National Academy of Sciences of the United States of America. 2019;116(13).

17. Singh P, Doti R, Lugo JE, Faubert J, Rawat S, Ghosh S, et al., editors. Biological infrared antenna and radar. Advances in Intelligent Systems and Computing; 2018.

18. Song $\mathrm{X}$, Zhang $\mathrm{F}, \mathrm{Bu} \mathrm{Y}$. Dynamic relaying properties of a $\beta$-turn peptide in long-range electron transfer. Journal of Computational Chemistry. 2019;40(9).

19. Kraus JD. Helical Beam Antennas for Wide-Band Applications. Proceedings of the IRE. 1948;36(10).

20. Wheeler HA. A helical antenna for circular polarization. Proceedings of the IRE. 1947;35(12).

21. Stilwell RK. Satellite applications of the bifilar helix antenna. Johns Hopkins APL Technical Digest (Applied Physics Laboratory). 1991;12(1).

22. Amin M, Cahill R. Side-fed bifilar helix antenna. IEEE microwave and wireless components letters. 2005;15(12):913-5.

23. Radman-Livaja M, Rando OJ. Nucleosome positioning: How is it established, and why does it matter? Developmental Biology2010.

24. Su C, Ke H, Hubing T, editors. A simplified model for normal mode helical antennas. ACES; 2010 .

25. Shokolenko IN, Alexeyev MF. Mitochondrial DNA: A disposable genome? Biochimica et Biophysica Acta - Molecular Basis of Disease2015.

26. Kilgus CC. Multielement, Fractional Turn Helices. IEEE Transactions on Antennas and Propagation. 1968;16(4).

27. Sexton T, Cavalli G. The role of chromosome domains in shaping the functional genome. Cell. 2015;160(6):1049-59. 
28. Lavelle C. Pack, unpack, bend, twist, pull, push: the physical side of gene expression. Current opinion in genetics \& development. 2014;25:74-84.

29. Smock RG, Gierasch LM. Sending signals dynamically. Science. 2009;324(5924):198-203.

30. Tompa P. The principle of conformational signaling. Chemical Society Reviews. 2016;45(15):4252-84.

31. Lupas AN, Bassler J. Coiled Coils - A Model System for the 21st Century. Trends in Biochemical Sciences2017.

32. Wong J, King H. Broadband quasi-taper helical antennas. IEEE Transactions on Antennas and Propagation. 1979;27(1):72-8.

33. Volakis JL. Antenna engineering handbook: McGraw-Hill Education; 2007.

34. Sing L, Mei K. Analysis of zigzag antennas. IEEE Transactions on Antennas and Propagation. 1970;18(6):760-4.

35. Sengupta D. The radiation characteristics of a zig-zag antenna. IRE Transactions on Antennas and Propagation. 1958;6(2):191-4.

36. Bhatnagar P, Sachan S. Analysis of infinite zig-zag antenna. IEE-IERE Proceedings-India. 1976;14(2):44-6.

37. Thiele GA, Ekelman EP. Design Formulas for Vee Dipoles. IEEE Transactions on Antennas and Propagation. 1980;28(4).

38. Barvitenko N, Lawen A, Aslam M, Pantaleo A, Saldanha C, Skverchinskaya E, et al. Integration of intracellular signaling: Biological analogues of wires, processors and memories organized by a centrosome 3D reference system. BioSystems. 2018;173.

39. Fletcher DA, Mullins RD. Cell mechanics and the cytoskeleton. Nature2010. p. 485-92.

40. Friesen DE, Craddock TJA, Kalra AP, Tuszynski JA. Biological wires, communication systems, and implications for disease. BioSystems2015.

41. Truebestein L, Leonard TA. Coiled-coils: The long and short of it. BioEssays. 2016;38(9).

42. Kamran M, Friebe VM, Delgado JD, Aartsma TJ, Frese RN, Jones MR. Demonstration of asymmetric electron conduction in pseudosymmetrical photosynthetic reaction centre proteins in an electrical circuit. Nature communications. 2015;6(1):1-9.

43. Bostick CD, Mukhopadhyay S, Pecht I, Sheves M, Cahen D, Lederman D. Protein bioelectronics: A review of what we do and do not know. Reports on Progress in Physics2018.

44. Dutour-Provenzano G, Etienne-Manneville S. Intermediate filaments. Current Biology. 2021;31(10):R522-R9.

45. Eldirany SA, Lomakin IB, Ho M, Bunick CG. Recent insight into intermediate filament structure. Current Opinion in Cell Biology2021.

46. Regolini M. The centrosome as a geometry organizer. Results and Problems in Cell Differentiation. 672019.

47. Gupta R, Goddard NJ. Leaky waveguides (LWs) for chemical and biological sensing-A review and future perspective. Sensors and Actuators, B: Chemical2020.

48. Jackson DR, Caloz C, Itoh T. Leaky-wave antennas. Proceedings of the IEEE. 2012;100(7):2194-206.

49. Bannister AJ, Kouzarides T. Regulation of chromatin by histone modifications. Cell Research2011.

50. Erler J, Zhang R, Petridis L, Cheng X, Smith JC, Langowski J. The Role of Histone Tails in the Nucleosome: A Computational Study. Biophysical Journal. 2014;107(12).

51. Maslovski S, Ikonen P, Kolmakov I, Tretyakov SA, Kaunisto M. Artificial magnetic materials based on the new magnetic particle: Metasolenoid. Progress in Electromagnetics Research. 2005;54.

52. Ball P. Water is an activematrix of life for cell and molecular biology. Proceedings of the National Academy of Sciences of the United States of America2017.

53. Bellissent-Funel MC, Hassanali A, Havenith M, Henchman R, Pohl P, Sterpone F, et al. Water Determines the Structure and Dynamics of Proteins. Chemical Reviews2016.

54. Wong KL, Hsiao FR, Chiou TW. Omnidirectional planar dipole array antenna. IEEE Transactions on Antennas and Propagation. 2004;52(2).

55. Li B, Liao SW, Xue Q. Omnidirectional circularly polarized antenna combining monopole and loop radiators. IEEE Antennas and Wireless Propagation Letters. 2013;12. 
56. Ahmad M, Gu W, Geyer T, Helms V. Adhesive water networks facilitate binding of protein interfaces. Nature Communications. 2011;2(1).

57. Kuffel A. How water mediates the long-range interactions between remote protein molecules. Physical Chemistry Chemical Physics. 2017;19(7).

58. Laage D, Elsaesser T, Hynes JT. Water Dynamics in the Hydration Shells of Biomolecules. Chemical Reviews. 2017;117(16).

59. Wei L, Rodríguez-Fortuño FJ. Far-field and near-field directionality in acoustic scattering. New Journal of Physics. 2020;22(8).

60. Wimmer M, Palma JL, Tarakeshwar P, Mujica V. Single-Molecule Conductance through Hydrogen Bonds: The Role of Resonances. Journal of Physical Chemistry Letters. 2016;7(15).

61. Nakano H, Iitsuka Y, Yamauchi J. Rhombic grid array antenna. IEEE Transactions on Antennas and Propagation. 2013;61(5).

62. Silberberg MS, Amateis P. Chemistry: The molecular nature of matter and change: Mosby St. Louis, Missouri, USA; 2021.

63. Gelbin A, Schneider B, Clowney L, Hsieh S-H, Olson WK, Berman HM. Geometric Parameters in Nucleic Acids: Sugar and Phosphate Constituents. Journal of the American Chemical Society. 1996;118(3):519-29.

64. Petosa A, Ittipiboon A, editors. Dielectric resonator antennas: A historical review and the current state of the art. IEEE Antennas and Propagation Magazine; 2010.

65. Lynch jr D. Stealth antennas and radomes. An Introduction to RF Stealth2021.

66. Auckland D, Daniel C, Diaz R, editors. A New type of conformal antenna using magnetic flux channels. Proceedings - IEEE Military Communications Conference MILCOM; 2014.

67. Luk KM, Wu B, editors. The magnetoelectric dipolea wideband antenna for base stations in mobile communications. Proceedings of the IEEE; 2012.

68. Erentok A, Ziolkowski RW. Metamaterial-inspired efficient electrically small antennas. IEEE Transactions on Antennas and Propagation. 2008;56(3).

69. Maksymov IS. Magneto-plasmonic nanoantennas: Basics and applications. Reviews in Physics2016.

70. Persch E, Dumele O, Diederich F. Molecular recognition in chemical and biological systems. Angewandte Chemie - International Edition2015.

71. Tononi G. Consciousness as integrated information: A provisional manifesto. Biological Bulletin: Marine Biological Laboratory; 2008. p. 216-42.

72. Bayne T, Hohwy J, Owen AM. Are There Levels of Consciousness? Trends in Cognitive Sciences2016.

73. Baars BJ, Edelman DB. Consciousness, biology and quantum hypotheses. Physics of Life Reviews. 2012;9(3):285-94.

74. Csermely P, Kunsic N, Mendik P, Kerestély M, Faragó T, Veres DV, et al. Learning of signaling networks: molecular mechanisms. Trends in biochemical sciences. 2020;45(4):284-94.

75. Cui S. The possible roles of water in the prebiotic chemical evolution of DNA. Physical Chemistry Chemical Physics. 2010;12(35).

76. Rice SH. A stochastic version of the Price equation reveals the interplay of deterministic and stochastic processes in evolution. BMC Evolutionary Biology. 2008;8(1).

77. Carvunis AR, Rolland T, Wapinski I, Calderwood MA, Yildirim MA, Simonis N, et al. Protogenes and de novo gene birth. Nature. 2012;487(7407).

78. Gregory TR. Understanding Natural Selection: Essential Concepts and Common Misconceptions. Evolution: Education and Outreach. 2009;2(2).

79. Balanis CA. Antenna Theory: Analysis and Design, Fourth Edition. John Wiley \& Sons, Inc. 2016.

80. Selvan KT, Janaswamy R. Fraunhofer and Fresnel Distances : Unified derivation for aperture antennas. IEEE Antennas and Propagation Magazine. 2017;59(4).

81. Schab K, Jelinek L, Capek M, Ehrenborg C, Tayli D, Vandenbosch GAE, et al. Energy Stored by Radiating Systems. IEEE Access. 2018;6.

82. Schmelcher P. Molecule formation in ultrahigh magnetic fields. Science2012.

83. Burke BF, Graham-Smith F, Wilkinson PN. An introduction to radio astronomy2019. 
84. Louis CK, Louarn P, Allegrini F, Kurth WS, Szalay JR. Ganymede-Induced Decametric Radio Emission: In Situ Observations and Measurements by Juno. Geophysical Research Letters. 2020;47(20).

85. Martos YM, Imai M, Connerney JEP, Kotsiaros S, Kurth WS. Juno Reveals New Insights Into Io-Related Decameter Radio Emissions. Journal of Geophysical Research: Planets. 2020;125(7).

86. Tavlayan A, Tekin B. Exact formulas for spherical photon orbits around Kerr black holes. Physical Review D. 2020;102(10).

87. Burlaga LF, Ness NF. Heliosheath magnetic fields between 104 and 113 AU in a region of declining speeds and a stagnation region. Astrophysical Journal. 2012;749(1).

88. Alaee R, Filter R, Lehr D, Lederer F, Rockstuhl C. A generalized Kerker condition for highly directive nanoantennas. Optics Letters. 2015;40(11).

89. Geffrin JM, García-Cámara B, Gómez-Medina R, Albella P, Froufe-Pérez LS, Eyraud C, et al. Magnetic and electric coherence in forward-and back-scattered electromagnetic waves by a single dielectric subwavelength sphere. Nature Communications. 2012;3.

90. Kerker M, Wang DS, Giles CL. Electromagnetic scattering by magnetic spheres. Journal of the Optical Society of America. 1983;73(6).

91. Thal HL. Gain and Q bounds for coupled TM-TE modes. IEEE Transactions on Antennas and Propagation. 2009;57(7).

92. Anderson NR, Camley RE. Multilayer magnetic waveguides: Optimizing nonreciprocal propagation in the 50-70GHz range. Journal of Applied Physics. 2014;116(2).

93. Giles CL, Wild WJ. Fresnel reflection and transmission at a planar boundary from media of equal refractive indices. Applied Physics Letters. 1982;40(3).

94. West J, Landecker T, Gaensler B, Jaffe T, Hill A. A Unified Model for the Fan Region and the North Polar Spur: A bundle of filaments in the Local Galaxy. arXiv preprint arXiv:210914720. 2021.

95. Libeskind NI, van de Weygaert R, Cautun M, Falck B, Tempel E, Abel T, et al. Tracing the cosmic web. Monthly Notices of the Royal Astronomical Society. 2017;473(1):1195-217.

96. Tang P, editor A design of high performance splash-plate feed for parabolic reflector antenna. 2017 IEEE 6th Asia-Pacific Conference on Antennas and Propagation, APCAP 2017 - Proceeding; 2018.

97. Selvaraja SK, Sethi P. Review on Optical Waveguides. Emerging Waveguide Technology2018.

98. Deng H, Mayer L, Helled R. Formation of intermediate-mass planets via magnetically controlled disk fragmentation. Nature Astronomy. 2021;5(5).

99. Kim OS. Low-Q electrically small spherical magnetic dipole antennas. IEEE Transactions on Antennas and Propagation. 2010;58(7).

100. Das S, Iyer AK. Design of a Highly Miniaturized, Inherently Matched, Spherical Folded Dipole Antenna and Evaluation of its Quality Factor. IEEE Transactions on Antennas and Propagation. 2021. 101. Morishita H. Modern small antennas2010.

102. Karilainen AO, Ikonen PMT, Simovski CR, Tretyakov SA. Choosing dielectric or magnetic material to optimize the bandwidth of miniaturized resonant antennas. IEEE Transactions on Antennas and Propagation. 2011;59(11).

103. Picardi MF, Zayats AV, Rodríguez-Fortuño FJ. Janus and Huygens Dipoles: Near-Field Directionality beyond Spin-Momentum Locking. Physical Review Letters. 2018;120(11).

104. Leonhardt U, Philbin TG. General relativity in electrical engineering. New Journal of Physics. $2006 ; 8$.

105. Costa LFO, Natário J. Gravito-electromagnetic analogies. General Relativity and Gravitation. 2014;46(10):1-57.

106. Genov DA. General relativity: Optical black-hole analogues. Nature Photonics. 2011;5(2).

107. Rosenberg Y. Optical analogues of black-hole horizons: Optical analogues. Philosophical Transactions of the Royal Society A: Mathematical, Physical and Engineering Sciences. 2020;378(2177).

108. Curto AG, Volpe G, Taminiau TH, Kreuzer MP, Quidant R, Van Hulst NF. Unidirectional emission of a quantum dot coupled to a nanoantenna. Science. 2010;329(5994). 
109. Eggleston MS, Messer K, Zhang L, Yablonovitch E, Wu MC. Optical antenna enhanced spontaneous emission. Proceedings of the National Academy of Sciences of the United States of America. 2015;112(6).

110. Giannini V, Fernández-Domínguez AI, Heck SC, Maier SA. Plasmonic nanoantennas: Fundamentals and their use in controlling the radiative properties of nanoemitters. Chemical Reviews2011.

111. Alexander S, Cunningham WJ, Lanier J, Smolin L, Stanojevic S, Toomey MW, et al. The Autodidactic Universe. arXiv preprint arXiv:210403902. 2021.

112. Mozneb M, Mirtaheri E, Sanabria AO, Li CZ. Bioelectronic properties of DNA, protein, cells and their applications for diagnostic medical devices. Biosensors and Bioelectronics2020.

113. Slepyan GY, Vlasenko S, Mogilevtsev D. Quantum Antennas. Advanced Quantum Technologies2020.

114. Cao J, Cogdell RJ, Coker DF, Duan HG, Hauer J, Kleinekathöfer U, et al. Quantum biology revisited. Science Advances. 2020;6(14).

115. Varshney LR, Sun JZ. Why do we perceive logarithmically? Significance. 2013;10(1):28-31.

116. Vallauri R, Bertin G, Piovano B, Gianola P. Electromagnetic Field Zones Around an Antenna for Human Exposure Assessment: Evaluation of the human exposure to EMFs. IEEE Antennas and Propagation Magazine. 2015;57(5).

117. Bandara $\mathrm{P}$, Carpenter DO. Planetary electromagnetic pollution: it is time to assess its impact. The Lancet Planetary Health2018.

118. Miller AB, Sears ME, Morgan LL, Davis DL, Hardell L, Oremus M, et al. Risks to health and well-being from radio-frequency radiation emitted by cell phones and other wireless devices. Frontiers in Public Health2019. 


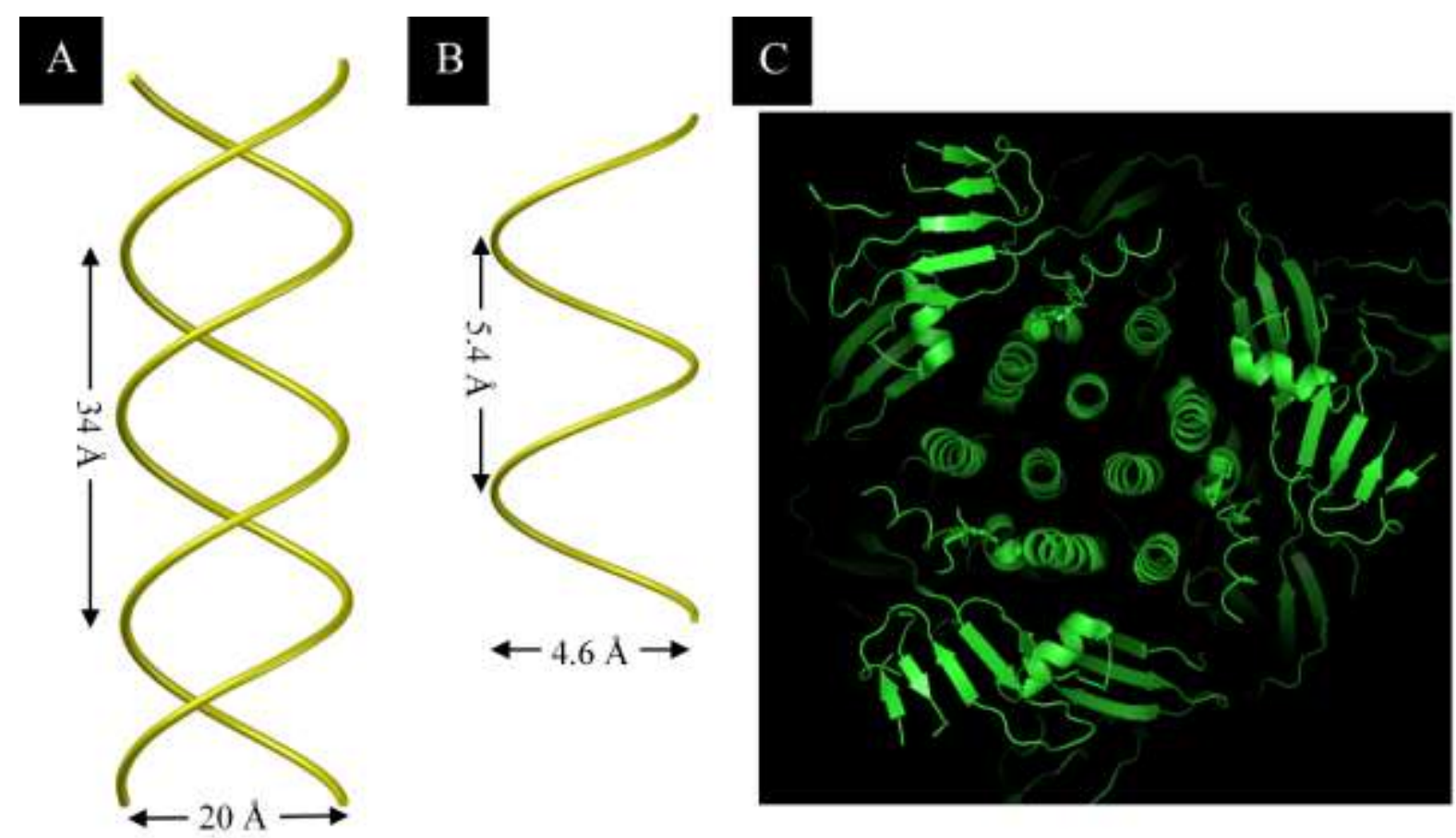

\section{D}
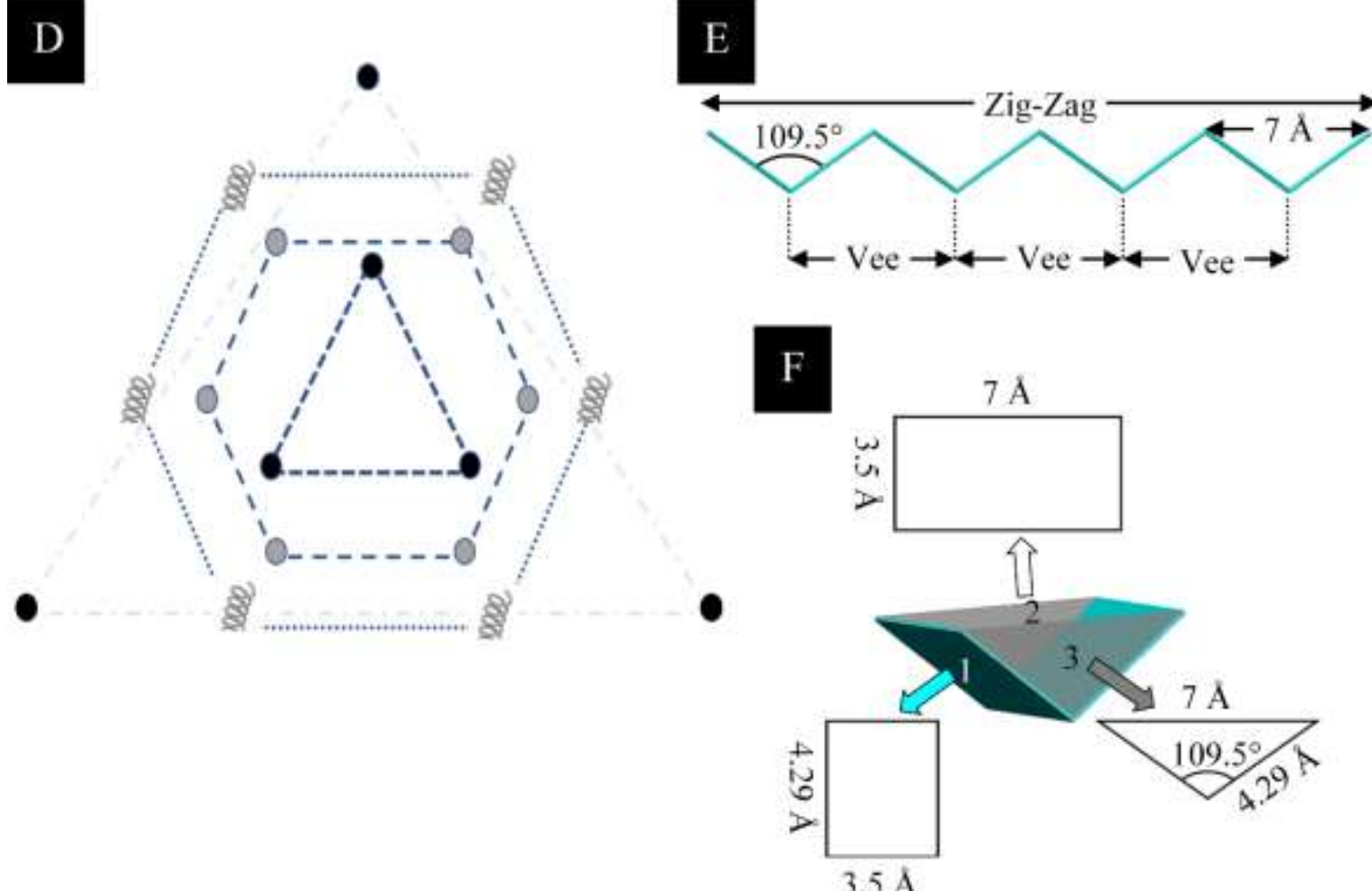

Figure 1 Illustrates the structural parameters of A) B-DNA, B) $\alpha$-helix, C) Arrangement of $\alpha$-helices in the spike protein of SARS-CoV-2 (PDB ID:7CAB), D) Hexagonal and triangular pattern of the $\alpha-$ helices in the spike protein, E) $\beta$-strand with zigzag and Vee-antenna configuration and F) Sides of an antiparallel- $\beta$-sheet unit. 


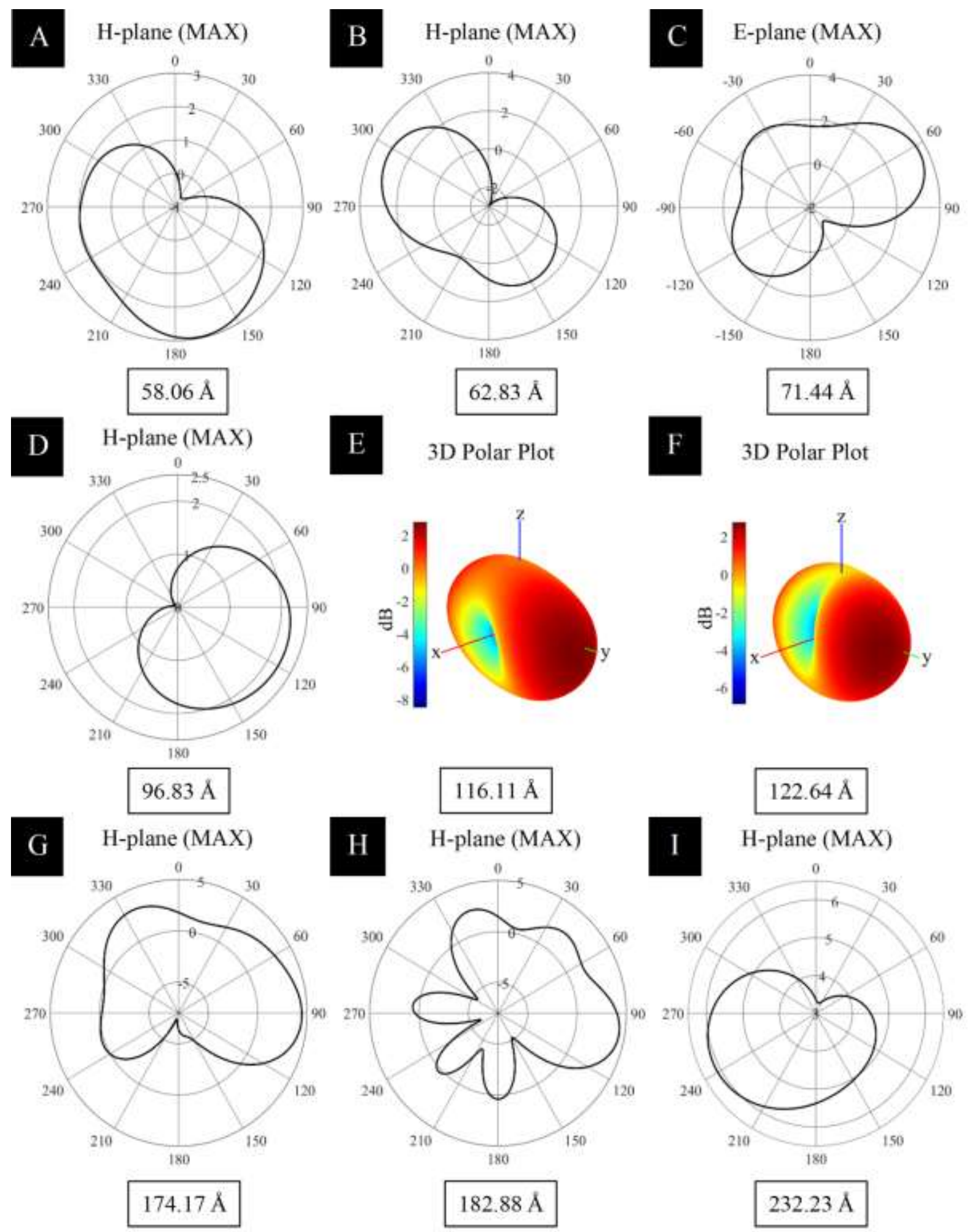

Figure 2 The radiation patterns of B-DNA at the different wavelengths of B-DNA. While (E) and (F) are the 3D polar plots at wavelengths $116.11 \AA$ and $122.64 \AA$, and $(\mathbf{A}),(\mathbf{B}),(\mathbf{C}),(\mathbf{D}),(\mathbf{G}),(\mathbf{H})$, and $(\mathbf{I})$ are the 2D radiation patterns of wavelengths $58.06 \AA, 62.83 \AA, 71.44 \AA, 96.83 \AA, 174.17 \AA, 182.88 \AA$, and $232.23 \AA$. Radiation patterns reveal that the wavelengths of B-DNA show cardioid patterns and mutual coupling effects. 

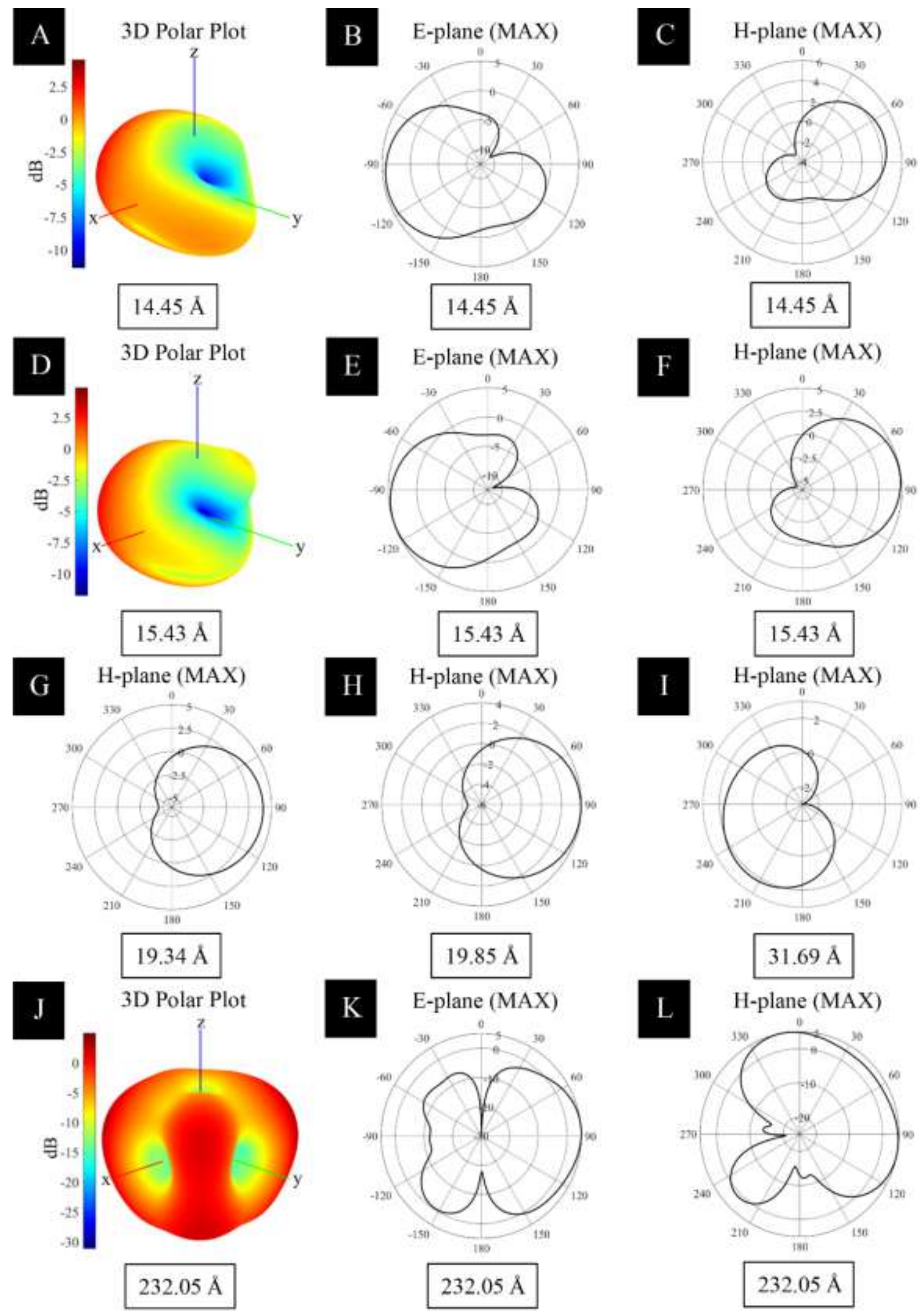

Figure 3 Illustrates the radiation patterns of $\alpha$-helix at the different wavelengths. (A), (D) and (J) are the 3D polar plots of $14.45 \AA, 15.43 \AA$ and $232.05 \AA$, and $(\mathbf{B}, \mathbf{C}),(\mathbf{E}, \mathbf{F}),(\mathbf{G}),(\mathbf{H}),(\mathbf{I})$ and $(\mathbf{K}, \mathbf{L})$ are the 2D radiation patterns of $14.45 \AA, 15.43 \AA, 19.34 \AA, 19.85 \AA, 31.69 \AA$ and $232.05 \AA$. The radiation patterns depict that the wavelengths of $\alpha$-helix exhibit cardioid and unidirectional radiation patterns. 


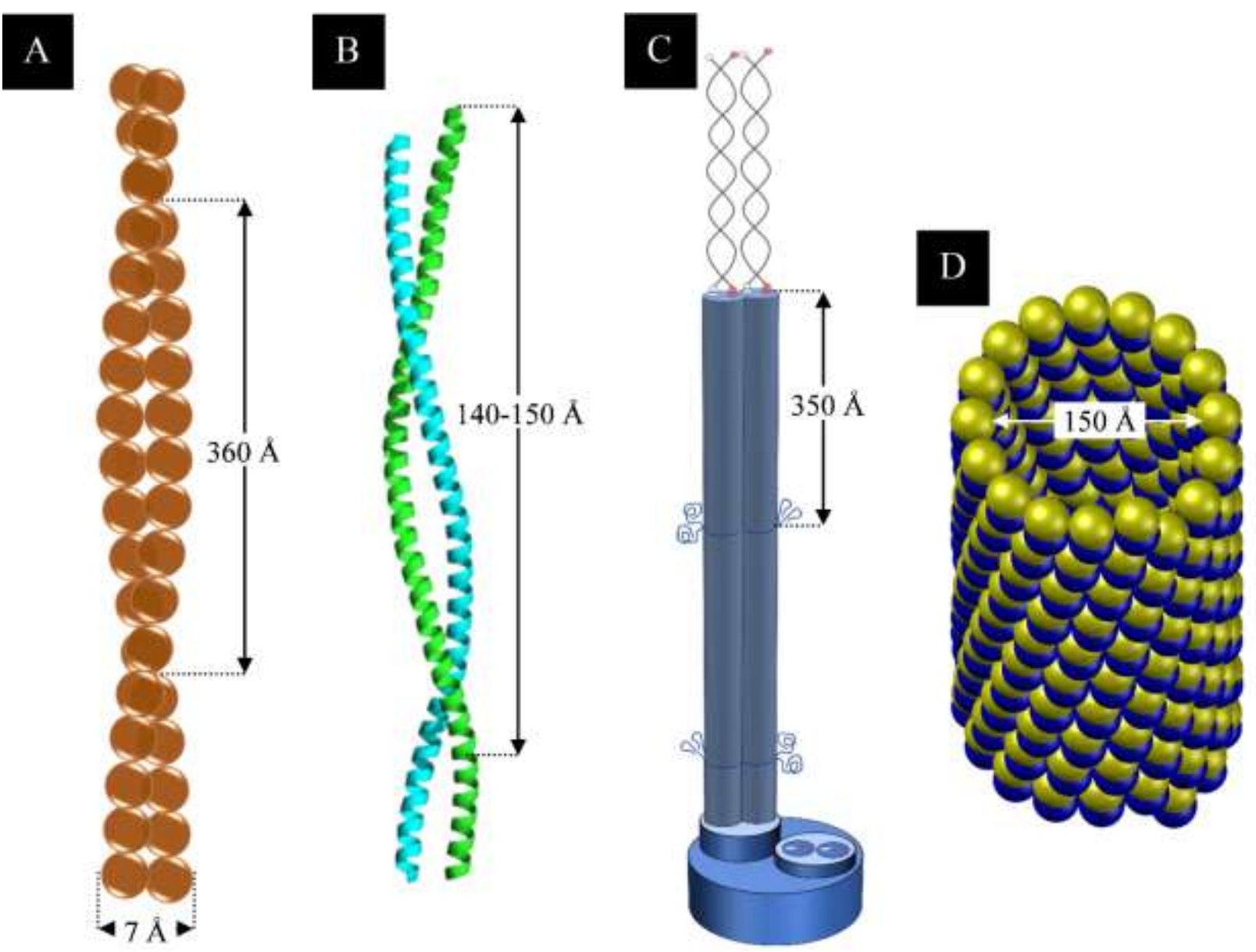

Figure 4 Depicts the structure of the cytoskeleton system, A) Microfilament, B) Extended coil-coiled structure, C) Protofibril of intermediate filament, and D) Microtubule. 


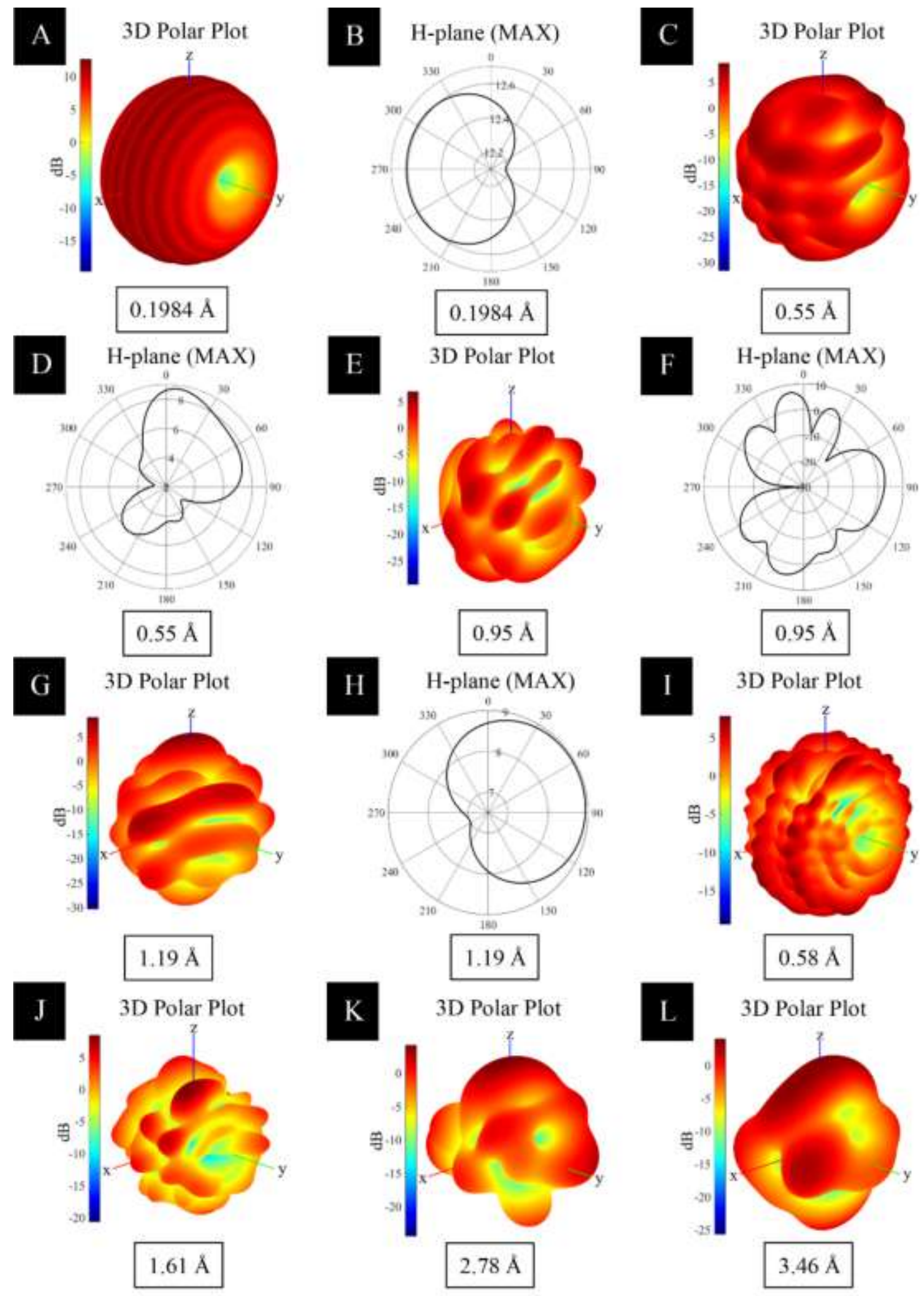

Figure 5 Depicts radiation patterns of a water molecule and water molecule associated with hydrogen bonds. (A), (C), (E), and (G) are the 3D polar plots of $0.1984 \AA, 0.55 \AA, 0.95 \AA$, and $1.19 \AA$ of water. (B), (D), (F), and (H) are the 2D radiation patterns of $0.1984 \AA, 0.55 \AA, 0.95 \AA$, and $1.19 \AA$ of water. $(\mathrm{I}),(\mathrm{J}),(\mathrm{K})$, and $(\mathrm{L})$ are the $3 \mathrm{D}$ polar plots of $0.58 \AA, 1.61 \AA, 2.78 \AA$, and $3.46 \AA$ of an H-bonded water molecule. The patterns reveal that water exhibits cardioid and omnidirectional array radiation patterns. 


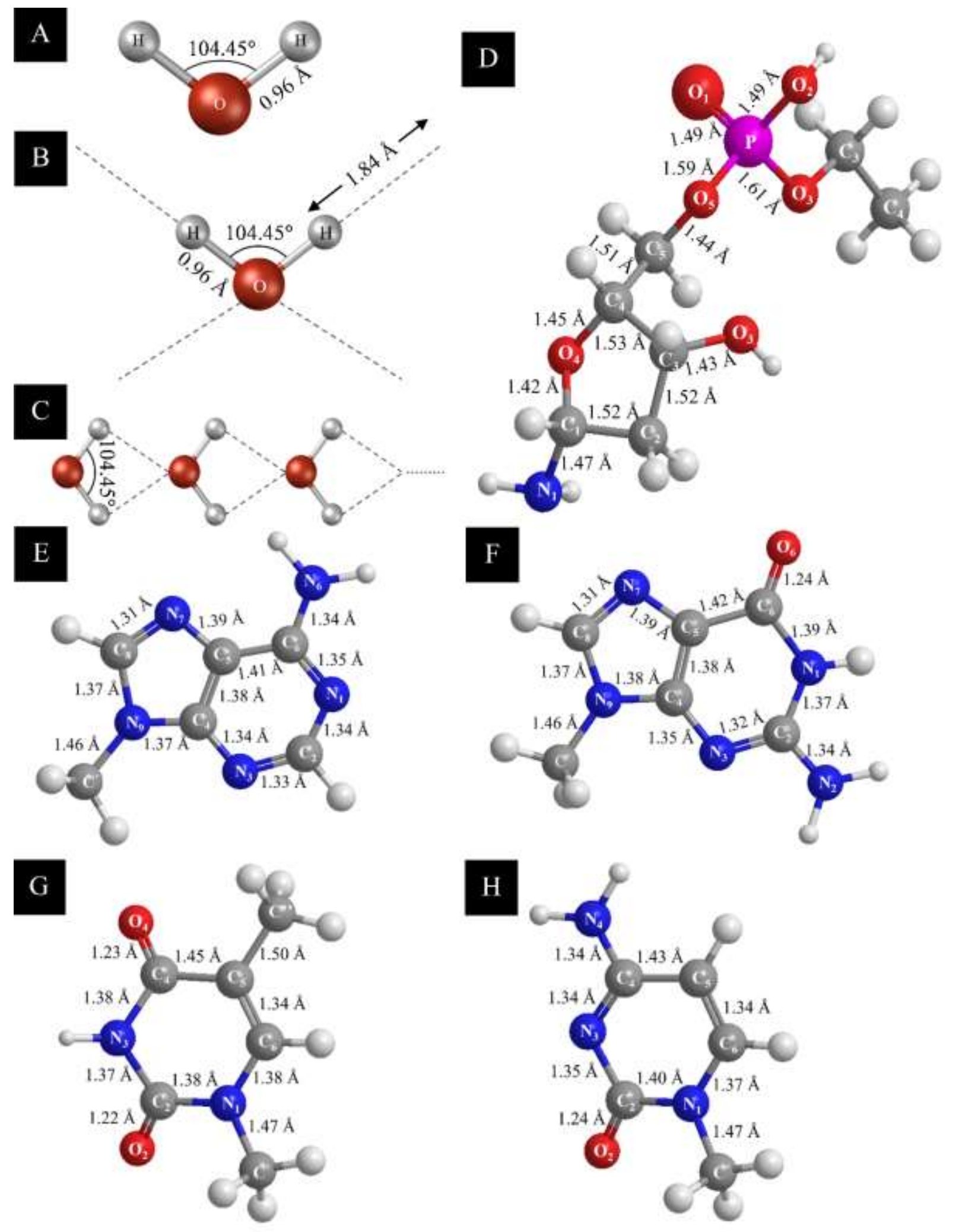

Figure 6 Illustrates the structures of A) Water molecule, B) Water molecule associated with hydrogen bonds, C) Rhombic configuration of water, D) ribose sugar, E) Adenine, F) Guanine, G) Thymine, and H) Cytosine. 
Table 1 The structural parameters, wavelengths, and wirelengths of biomolecules considered for the study.

\begin{tabular}{|c|c|c|c|c|c|c|c|}
\hline $\begin{array}{c}\text { Sl. } \\
\text { No. }\end{array}$ & \multicolumn{2}{|c|}{ Parameters } & \multirow{3}{*}{$\begin{array}{c}\text { Wavelength }(\AA) \\
34 \\
20\end{array}$} & \multirow{3}{*}{$\begin{array}{l}\text { Sl. } \\
\text { No. } \\
41 . \\
42 .\end{array}$} & \multicolumn{2}{|c|}{ Parameters } & \multirow{3}{*}{$\begin{array}{c}\text { Wavelength }(\mathbf{\AA}) \\
4.2858 \\
7\end{array}$} \\
\hline 1. & & Pitch & & & \multirow{9}{*}{$\begin{array}{l}\vec{d} \\
\frac{d}{\omega} \\
\qquad \\
\varrho\end{array}$} & Arm length & \\
\hline 2. & Structure & Diameter & & & & Aperture & \\
\hline 3. & \multirow{6}{*}{ Wavelength } & Axial & 62.83185 & 43. & & Distance $^{3}$ & 3.5 \\
\hline 4. & & & 58.05650 & 44. & & \multirow{6}{*}{ Wavelength } & 0.86882 \\
\hline 5. & & & 116.11299 & 45. & & & 2.41857 \\
\hline 6. $\longleftarrow$ & & Normal & 174.16949 & 46. & & & 4.69702 \\
\hline 7. 台 & & & 232.22599 & 47. & & & 5.54715 \\
\hline 8. & & $\mathrm{HTBH}^{1}$ & 182.88235 & 48. & & & 1.57169 \\
\hline $\begin{array}{r}9 . \\
10\end{array}$ & $\mathrm{SRW}^{2}$ & $\begin{array}{l}\text { Turn } 1 \\
\text { Turn } 2\end{array}$ & $\begin{array}{l}122.64407 \\
208.67579\end{array}$ & $\begin{array}{l}49 . \\
50 .\end{array}$ & & & $\begin{array}{l}5.71649 \\
3.81099\end{array}$ \\
\hline 10. & \multirow[b]{2}{*}{ Wirelength } & $\begin{array}{l}\text { Standard } \\
\text { Stal } 2 \\
\end{array}$ & $\frac{200.0 / 519}{71.44118}$ & 51. & \multirow{5}{*}{ 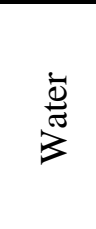 } & Arm length & 0.96 \\
\hline 12. & & Small & 96.83185 & 52. & & \multirow{4}{*}{ Wavelength } & 0.19844 \\
\hline 13. & \multirow{2}{*}{ Structure } & Pitch & 5.4 & 53. & & & 0.55298 \\
\hline 14. & & Diameter & 4.6 & 54. & & & 0.95277 \\
\hline 15. & \multirow{14}{*}{ Wavelength } & Axial & 14.45133 & $\frac{55 .}{56}$ & & & 1.18575 \\
\hline 16. & & \multirow{13}{*}{ Normal } & 19.33711 & 56. & & Arm length & 2.8 \\
\hline 17. & & & 38.67423 & & $\frac{\tilde{D}}{\tilde{\sigma}}$ & & 0.57877 \\
\hline 18. & & & 58.01134 & 58. & हี & Wavelength & 2.77891 \\
\hline 19. & & & 77.34846 & 59. & $I$ & & 1.61287 \\
\hline 20. & & & 96.68557 & 60. & & & 3.45843 \\
\hline 21. & & & 116.02268 & & & & \\
\hline 22. & & & 135.35980 & & & & \\
\hline 23. & & & 154.69691 & & & & \\
\hline 24. & & & 174.03402 & & & & \\
\hline 25. & & & 193.37114 & & & & \\
\hline 26. $: \frac{x}{\theta}$ & & & 212.70825 & & & & \\
\hline 27. $\frac{8}{\frac{1}{x}}$ & & & 232.04537 & & & & \\
\hline 28 & & & 251.38248 & & & & \\
\hline 29. & \multirow{10}{*}{ SRW2 } & Turn 1 & 31.68575 & & & & \\
\hline 30. & & Turn 2 & 51.86493 & & & & \\
\hline 31. & & Turn 3 & 71.53494 & & & & \\
\hline 32. & & Turn 4 & 91.05494 & & & & \\
\hline 33. & & Turn 5 & 110.51048 & & & & \\
\hline 34. & & Turn 6 & 129.93244 & & & & \\
\hline 35. & & Turn 7 & 149.33468 & & & & \\
\hline 36. & & Turn 8 & 168.72436 & & & & \\
\hline 37. & & Turn 9 & 188.10554 & & & & \\
\hline 38. & & Turn 10 & 207.48071 & & & & \\
\hline 39. & \multirow{2}{*}{ Wirelength } & Standard & 15.42728 & & & & \\
\hline 40. & & Small & 19.85133 & & & & \\
\hline
\end{tabular}

\footnotetext{
${ }^{1}$ HTBH: Half Turn Bifilar Helical Antenna

${ }^{2}$ SRW: Self-Resonant Wavelength

${ }^{3}$ Distance between two consecutive $\beta$-strand
} 
Table 2 Relationships between B-DNA and $\alpha$-helix

\begin{tabular}{cccccc}
\hline SI. No. & $\begin{array}{c}\text { Antenna } \\
\text { Parameters }(\AA)\end{array}$ & Relationship $(\AA)$ & $\begin{array}{c}\text { Antenna } \\
\text { Parameters }(\AA)\end{array}$ & Ratio & $\begin{array}{c}\text { Percentage } \\
\text { difference }(\%)\end{array}$ \\
\hline 1. & 58.06 & $58.06 \approx 19.34 \times 3$ & 19.34 & $3: 1$ & 0.07783 \\
2. & 96.83 & $96.83 \approx 19.34 \times 5$ & 19.34 & $5: 1$ & 0.15120 \\
3. & 58.06 & $58.06 \approx 14.45 \times 4$ & 14.45 & $4: 1$ & 0.43359 \\
4. & 19.34 & $19.34 \times 4 \approx 15.43 \times 5$ & 15.43 & $5: 4$ & 0.27451 \\
5. & 62.83 & $62.83 \approx 31.69 \times 2$ & 31.69 & $2: 1$ & 0.85521 \\
6. & 135.36 & $135.36 \approx 34 \times 4$ & 34.00 & $4: 1$ & 0.47187 \\
7. & 58.01 & $58.01 \approx 14.45 \times 4$ & 14.45 & $4: 1$ & 0.35575 \\
8. & 212.71 & $212.71 \approx 71.44 \times 3$ & 71.44 & $3: 1$ & 0.75654 \\
9. & 251.38 & $251.38 \approx 62.83 \times 4$ & 62.83 & $4: 1$ & 0.02189 \\
10. & 182.88 & $182.88 \approx 58.06 \times \pi$ & 58.06 & $\pi: 1$ & 0.26965 \\
11. & 62.83 & $62.83 \approx 19.85 \times \pi$ & 19.85 & $\pi: 1$ & 0.74612 \\
12. & 96.83 & $96.83 \approx 15.43 \times 2 \pi$ & 15.43 & $2 \pi: 1$ & 0.10385 \\
13. & 34.00 & $34 \approx 5.4 \times 2 \pi$ & 5.40 & $2 \pi: 1$ & 0.20845 \\
14. & 19.34 & $19.34 \times 5 \approx 15.43 \times 2 \pi$ & 15.43 & $2 \pi: 5$ & 0.25505 \\
15. & 71.44 & $71.44 \approx 19.85 \times 3.6$ & 19.85 & $3.6: 1$ & 0.03304 \\
16. & 19.34 & $19.34 \approx 5.4 \times 3.6$ & 5.40 & $3.6: 1$ & 0.53067 \\
17. & 5.40 & $5.4 \times 4 \approx 4.29 \times 5$ & $4.29(15.43 / 3.6)$ & $5: 4$ & 0.80518 \\
18. & 34.00 & $34 \approx 6.80 \times 5$ & $6.80(71.44 / 10.5)$ & $5: 1$ & 0.05766 \\
19. & 5.40 & $5.4 \times 5=6.80 \times 4$ & $6.80(71.44 / 10.5)$ & $4: 5$ & 0.79566 \\
20. & 58.06 & $58.06 \approx 9.22 \times 2 \pi$ & $9.22(96.83 / 10.5)$ & $2 \pi: 1$ & 0.19389 \\
21. & $9.22(96.83 / 10.5)$ & $9.22 \times 3 \approx 5.53 \times 5$ & $5.53(58.06 / 10.5)$ & $5: 3$ & 0.07337 \\
\hline
\end{tabular}


Table 3 The maximum antenna gain of B-DNA, $\alpha$-helix, water, and H-bonded water molecule as transceiver system.

\begin{tabular}{|c|c|c|c|c|c|}
\hline \multirow{2}{*}{ Sl. No. } & \multirow{2}{*}{$\begin{array}{c}\text { Wavelength } \\
\text { (A) }\end{array}$} & \multicolumn{4}{|c|}{ Maximum Gain (dB) } \\
\hline & & B-DNA & $\alpha$-helix & Water & H-bonded water \\
\hline 1. & 58.06 & 2.97 & 1.88 & 1.84 & 1.91 \\
\hline 2. & 62.83 & 3.08 & 1.95 & 1.87 & 1.91 \\
\hline 3. & 71.44 & 3.63 & 1.86 & 1.92 & 1.86 \\
\hline 4. & 96.83 & 2.23 & 1.83 & 1.91 & 1.85 \\
\hline 5. & 116.11 & 2.80 & 1.90 & 1.85 & 1.93 \\
\hline 6. & 122.64 & 2.77 & 1.95 & 1.90 & 1.89 \\
\hline 7. & 174.17 & 4.47 & 1.91 & 1.86 & 1.89 \\
\hline 8. & 182.88 & 4.41 & 1.82 & 1.90 & 1.93 \\
\hline 9. & 232.23 & 6.11 & 4.93 & 5.25 & 5.19 \\
\hline 10. & 14.45 & & 4.55 & 1.92 & 1.77 \\
\hline 11. & 15.43 & & 4.92 & 1.88 & 1.88 \\
\hline 12. & 19.34 & & 4.08 & 1.93 & 1.88 \\
\hline 13. & 19.85 & & 3.95 & 1.87 & 1.83 \\
\hline 14. & 31.69 & & 2.23 & 1.84 & 1.96 \\
\hline 15. & 38.67 & & 2.05 & 1.95 & 1.91 \\
\hline 16. & 58.01 & & 1.94 & 1.90 & 2.00 \\
\hline 17. & 77.35 & & 1.82 & 1.96 & 1.87 \\
\hline 18. & 96.69 & & 1.98 & 1.86 & 1.94 \\
\hline 19. & 116.02 & & 1.86 & 1.95 & 1.92 \\
\hline 20. & 135.36 & & 1.90 & 1.86 & 1.89 \\
\hline 21. & 154.70 & & 1.96 & 1.86 & 1.85 \\
\hline 22. & 174.03 & & 1.87 & 1.97 & 1.89 \\
\hline 23. & 193.37 & & 1.89 & 1.90 & 1.82 \\
\hline 24. & 212.71 & & 1.82 & 1.94 & 1.82 \\
\hline 25. & 232.05 & & 4.98 & 5.19 & 5.17 \\
\hline 26. & 251.38 & & 5.33 & 5.13 & 5.10 \\
\hline 27. & 0.1984 & & & 12.61 & \\
\hline 28. & 0.55 & & & 8.75 & \\
\hline 29. & 0.95 & & & 6.87 & \\
\hline 30. & 1.19 & & & 8.96 & \\
\hline 31. & 0.58 & & & & 7.78 \\
\hline 32. & 1.61 & & & & 8.48 \\
\hline 33. & 2.78 & & & & 4.25 \\
\hline 34. & 3.46 & & & & 4.07 \\
\hline
\end{tabular}


Table 4 Relationships of $\beta$-strands/ $\beta$-sheets with B-DNA and $\alpha$-helix

\begin{tabular}{|c|c|c|c|c|}
\hline SI.No. & $\begin{array}{c}\text { Antenna parameters of } 1 \\
\beta \text {-strands/ } \beta \text {-sheets }\end{array}$ & $\begin{array}{l}\text { Relation } \\
\text { ship }\end{array}$ & $\begin{array}{l}\text { Antenna parameters of other } \\
\text { biomolecules }\end{array}$ & $\begin{array}{c}\text { Percentage } \\
\text { difference (\%) }\end{array}$ \\
\hline 1. & $\begin{array}{c}\text { Arms of } \beta \text {-strand } / \beta \text {-sheet } \\
=4.286 \AA\end{array}$ & $\approx$ & $\begin{array}{l}\text { Length of each amino-acids of } \alpha \text {-helix } \\
(15.43 \AA / 3.6=4.285 \AA)\end{array}$ & 0.01145 \\
\hline 2. & $\begin{array}{c}\text { Zig-zag antenna } \\
\text { wavelength }=1.57 \AA\end{array}$ & $\approx$ & $\begin{array}{c}\text { 1/4 of effective length }(6.32 \AA) \text { of } \\
\text { wavelength } 19.85 \AA\end{array}$ & 0.50953 \\
\hline 3. & $\begin{array}{c}\text { V-antennae wavelength }= \\
5.55 \AA\end{array}$ & $\approx$ & $\begin{array}{l}\text { Length of each amino acid of small } \alpha \text {-helix } \\
\qquad(19.85 \AA / 3.6=5.51 \AA)\end{array}$ & 0.59471 \\
\hline 4. & $\begin{array}{c}\text { V-antennae wavelength }= \\
0.87 \AA\end{array}$ & $\approx$ & $\begin{array}{c}\text { 1/200 of normal mode wavelength of } \alpha- \\
\text { helix (174.03 } \AA \text { ) }\end{array}$ & 0.15526 \\
\hline 5. & $\begin{array}{c}\text { V-antennae wavelength }= \\
4.70 \AA\end{array}$ & $\approx$ & $\begin{array}{c}\text { 1/50 of normal mode wavelength of B- } \\
\text { DNA }(232.23 \AA)\end{array}$ & 1.20184 \\
\hline 6. & $\begin{array}{c}\text { V-antennae wavelength }= \\
2.42 \AA\end{array}$ & $\approx$ & $\begin{array}{c}1 / 10 \text { of the physical length } 24.21 \AA \text { for } \\
\text { effective length } 15.43 \AA\end{array}$ & 0.10041 \\
\hline 7. & $\begin{array}{l}\text { Horn antenna wavelength } \\
\text { in E-field }=5.72 \AA\end{array}$ & $\approx$ & $\begin{array}{c}\text { 1/4 of effective length }(11.37 \AA) \text { of } \\
\text { wavelength } 71.44 \AA\end{array}$ & 0.55048 \\
\hline 8. & $\begin{array}{l}\text { Horn antenna wavelength } \\
\quad \text { in } \mathrm{H} \text {-field }=3.81 \AA\end{array}$ & $\approx$ & 4 times the wavelength of water $(0.95 \AA)$ & 0.00236 \\
\hline 9. & $\begin{array}{c}\text { Rectangle sides: } 3.5 \AA \text {, } \\
4.29 \AA \text { A; Perimeter }= \\
15.57 \AA\end{array}$ & $\approx$ & Wire length of $\alpha$-helix (15.43 $\AA$ ) & 0.93172 \\
\hline 10. & $\begin{array}{l}\text { Rectangle sides: } 3.5 \AA \text {, } \\
4.29 \AA \text {; Perimeter of the } \\
\text { equiareal square }=15.49 \\
\AA\end{array}$ & $\approx$ & Wire length of $\alpha$-helix (15.43 Å) & 0.41975 \\
\hline 11. & $\begin{array}{l}\text { Rectangle sides: } 7 \AA, 3.5 \\
\AA \text { 我 Perimeter }=21 \AA\end{array}$ & $\approx$ & $\begin{array}{l}1 / 3 \text { of the axial wavelength of B-DNA } \\
\qquad(62.83 \AA)\end{array}$ & 0.26726 \\
\hline 12. & $\begin{array}{c}\text { Rectangle sides: } 7 \AA \text {, } 3.5 \\
\AA \text {; Perimeter of the } \\
\text { equiareal square }=19.80 \\
\AA\end{array}$ & $\approx$ & Wire length of small $\alpha$-helix (19.85 $\AA$ ) & 0.26401 \\
\hline 13. & $\begin{array}{c}\text { Triangle side: } 4.29 \AA \text { A } \\
4.29 \AA \text { A } 7 \AA \text { A } \text { Perimeter }= \\
15.57 \AA\end{array}$ & $\approx$ & Wire length of $\alpha$-helix (15.43 $\AA$ ) & 0.93172 \\
\hline
\end{tabular}


Table 5 Relationships of the water molecule with the antenna parameters of biomacromolecules

\begin{tabular}{|c|c|c|c|c|}
\hline Sl.No. & $\begin{array}{l}\text { Antenna parameters } \\
\text { of the water molecule }\end{array}$ & Relationship & $\begin{array}{l}\text { Antenna parameters of B- } \\
\text { DNA/ } \alpha \text {-helix/ } \beta \text {-strand }\end{array}$ & $\begin{array}{c}\text { Percentage } \\
\text { difference } \\
(\%) \\
\end{array}$ \\
\hline 1. & Arms of $\mathrm{H}_{2} \mathrm{O}=0.96 \AA$ & $\approx$ & $\begin{array}{l}1 / 100 \text { of the wire length of a } \\
\text { small B-DNA antenna ( } 96.83 \\
\AA \text { ) }\end{array}$ & 0.86277 \\
\hline 2. & $\begin{array}{c}\text { Length of Hydrogen } \\
\text { bonds }=1.84 \AA\end{array}$ & $\approx$ & $\begin{array}{c}\text { 1/100 of the wavelength of } \\
\text { B-DNA }(182.88 \AA)\end{array}$ & 0.60927 \\
\hline 3. & $\begin{array}{c}\text { Wavelength of Vee- } \\
\text { antenna }=1.19 \AA\end{array}$ & $\approx$ & 1/100 of (19.85 ̊×6) & 0.44848 \\
\hline 4. & $\begin{array}{l}\text { Wavelength of Vee- } \\
\text { antenna }=0.1984 \AA\end{array}$ & $\approx$ & $\begin{array}{c}1 / 100 \text { of the wire-length of } \\
\text { small } \alpha \text {-helix antenna (19.85 } \\
\AA \text { A) }\end{array}$ & 0.03693 \\
\hline 5. & $\begin{array}{l}\text { Wavelength of Vee- } \\
\text { antenna }=0.95 \AA\end{array}$ & $\approx$ & $\begin{array}{l}1 / 4 \text { of Horn antenna } \\
\text { wavelength in H-field of } \beta \text { - } \\
\text { sheet }(3.81 \AA)\end{array}$ & 0.00236 \\
\hline 6. & $\begin{array}{l}\text { Wavelength of Vee- } \\
\text { antenna }=0.55 \AA\end{array}$ & $\approx$ & $\begin{array}{l}1 / 10 \text { of the wavelength of } \beta- \\
\text { strand Vee antenna ( } 5.55 \AA)\end{array}$ & 0.31326 \\
\hline 7. & $\begin{array}{l}\text { Wavelength of Vee- } \\
\text { antenna }=0.55 \AA\end{array}$ & $\approx$ & $\begin{array}{l}\text { 1/10 of the length of each } \\
\text { amino acid of small } \alpha \text {-helix } \\
(19.85 \AA / 3.6=5.51 \AA)\end{array}$ & 0.28145 \\
\hline 8. & $\begin{array}{l}\text { Wavelength of }\left(\mathrm{H}_{2} \mathrm{O}+\right. \\
\text { Hydrogen bonds }) \text { as } \\
\text { Vee-antenna }=3.46 \AA\end{array}$ & $\approx$ & $1 / 50$ of $174 \AA(19.34 \AA \times 9)$ & 0.71912 \\
\hline 9. & $\begin{array}{l}\text { Wavelength of }\left(\mathrm{H}_{2} \mathrm{O}+\right. \\
\text { Hydrogen bonds }) \text { as } \\
\text { Vee-antenna }=0.58 \AA\end{array}$ & $\approx$ & $\begin{array}{l}1 / 100 \text { of the normal } \\
\text { wavelength of B-DNA } \\
(58.06 \AA)\end{array}$ & 0.30966 \\
\hline 10. & $\begin{array}{l}\text { Wavelength of }\left(\mathrm{H}_{2} \mathrm{O}+\right. \\
\text { Hydrogen bonds) as } \\
\text { Vee-antenna }=2.78 \AA\end{array}$ & $\approx$ & $\begin{array}{l}1 / 2 \text { wavelength of } \beta \text {-strand } \\
\text { Vee antenna }(5.55 \AA)\end{array}$ & 0.19217 \\
\hline 11. & $\begin{array}{l}\text { Wavelength of }\left(\mathrm{H}_{2} \mathrm{O}+\right. \\
\text { Hydrogen bonds }) \text { as } \\
\text { Vee-antenna = } 1.61 \AA\end{array}$ & $\approx$ & $1 / 2$ of $(34 \AA / 10.5)$ & 0.38229 \\
\hline 12. & $\begin{array}{l}\text { Wavelength of }\left(\mathrm{H}_{2} \mathrm{O}+\right. \\
\text { Hydrogen bonds) as } \\
\text { Vee-antenna }=1.61 \AA\end{array}$ & $\approx$ & 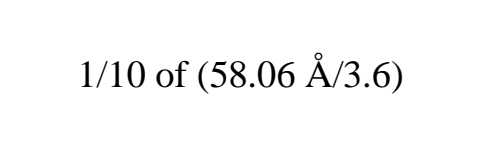 & 0.01175 \\
\hline 13. & $\begin{array}{c}\text { Circle with radius as } \\
0.96 \AA \text { A Perimeter of } \\
\text { the equiareal square = } \\
6.8 \AA\end{array}$ & $\approx$ & $\begin{array}{l}\text { Length of a nucleic acid of } \\
\text { B-DNA }(71.44 \AA / 10.5)\end{array}$ & 0.03381 \\
\hline 14. & $\begin{array}{c}\text { Circle with radius as } \\
2.8 \AA \text { 跬 Perimeter of } \\
\text { the equiareal square = } \\
19.85 \AA\end{array}$ & $\approx$ & $\begin{array}{l}\text { Wirelength of small } \alpha \text {-helix } \\
(19.85 \AA)\end{array}$ & 0.00077 \\
\hline
\end{tabular}


Table 6 Relationships among the chemical bonds and antenna parameters of biomolecules

\begin{tabular}{|c|c|c|c|c|c|}
\hline $\begin{array}{l}\text { Sl. } \\
\text { No. }\end{array}$ & Bonds & $\begin{array}{c}\begin{array}{c}\text { Average Bond } \\
\text { lengths }(\AA)\end{array} \\
\end{array}$ & Relationship & $\begin{array}{c}\text { Antenna parameters of } \\
\text { Biomolecules }(\AA)\end{array}$ & $\begin{array}{c}\text { Percentage } \\
\text { difference }(\%)\end{array}$ \\
\hline 1. & $\mathrm{C}-\mathrm{C}$ & 1.54 & $\approx$ & $\begin{array}{l}\text { 1/10 of the wirelength of } \alpha \text {-helix } \\
(15.43 \AA)\end{array}$ & 0.17699 \\
\hline 2. & $\mathrm{C}-\mathrm{O}$ & 1.43 & $\approx$ & $\begin{array}{l}\text { 1/100 of the wirelength of two } \\
\text { turns of B-DNA }(142.88 \AA)\end{array}$ & 0.08230 \\
\hline 3. & $\mathrm{C}-\mathrm{N}$ & 1.47 & $\approx$ & $\begin{array}{c}1 / 10 \text { of the axial mode } \\
\text { wavelength of } \alpha \text {-helix }(14.45 \AA)\end{array}$ & 1.70606 \\
\hline 4. & $\mathrm{C}-\mathrm{S}$ & 1.81 & $\approx$ & $\begin{array}{l}1 / 100 \text { of the wavelength of B- } \\
\text { DNA }(182.88 \AA)\end{array}$ & 1.03459 \\
\hline 5. & $\mathrm{C}-\mathrm{H}$ & 1.09 & $\approx$ & $1 / 100$ of $(15.43 \AA \times 7)$ & 0.93003 \\
\hline 6. & $\mathrm{~N}-\mathrm{H}$ & 1.01 & $\approx$ & $1 / 100$ of $(14.45 \AA \times 7)$ & 0.15761 \\
\hline 7. & $\mathrm{~N}-\mathrm{O}$ & 1.44 & $\approx$ & $\begin{array}{l}1 / 10 \text { of the axial mode } \\
\text { wavelength of } \alpha \text {-helix }(14.45 \AA)\end{array}$ & 0.35582 \\
\hline 8. & S-S & 2.04 & $\approx$ & $1 / 100$ of $(15.43 \AA \times 13)$ & 1.70329 \\
\hline 9. & $\mathrm{~S}-\mathrm{H}$ & 1.34 & $\approx$ & $\begin{array}{c}1 / 100 \text { of }(26.90 \AA \times 5)--96.83 \AA \\
/ 3.6\end{array}$ & 0.36402 \\
\hline 10. & $\mathrm{C}=\mathrm{C}$ & 1.34 & $\approx$ & $\begin{array}{c}1 / 100 \text { of }(26.90 \AA \times 5)--96.83 \AA \\
/ 3.6\end{array}$ & 0.36402 \\
\hline 11. & $\mathrm{C}=\mathrm{O}$ & 1.23 & $\approx$ & $\begin{array}{c}1 / 100 \text { of the self-resonant } \\
\text { wavelength of B-DNA (122.64 } \\
\AA \text { A })\end{array}$ & 0.28979 \\
\hline 12. & $\mathrm{C}=\mathrm{O}$ & 1.23 & $\approx$ & $1 / 100$ of $(15.43 \AA ̊ 8)$ & 0.33946 \\
\hline 13. & $\mathrm{C}=\mathrm{N}$ & 1.27 & $\approx$ & $1 / 100$ of $(31.69 \AA \times 4)$ & 0.20257 \\
\hline 14. & $\mathrm{~N}=\mathrm{O}$ & 1.20 & $\approx$ & $1 / 100$ of $(19.85 \AA \times 6)$ & 0.74612 \\
\hline 15. & $\mathrm{PO}_{4}$ & 1.54 & $\approx$ & $\begin{array}{l}\text { 1/10 of the wirelength of } \alpha \text {-helix } \\
(15.43 \AA)\end{array}$ & 0.17699 \\
\hline 16. & $\mathrm{P}-\mathrm{O}$ & 1.60 & $\approx$ & $1 / 100$ of $19.85 \AA \times 8 \approx 160 \AA$ & 0.74612 \\
\hline 17. & $\mathrm{P}=\mathrm{O}$ & 1.48 & $\approx$ & $1 / 100$ of $9.22 \AA \times 16 \approx 148 \AA$ & 0.30228 \\
\hline
\end{tabular}


Table 7 The wavelength and Reactive Near Field Radius (ReNFR) for planets of the solar system considering Einstein's general relativity and Kepler's $3^{\text {rd }}$ law of planetary motion.

\begin{tabular}{|c|c|c|c|c|c|c|c|}
\hline $\begin{array}{l}\text { Sl. } \\
\text { No. }\end{array}$ & Planets & $\begin{array}{c}\text { Polar } \\
\text { Diameter } \\
(\mathbf{m})\end{array}$ & $\begin{array}{l}\text { Schwarzschild } \\
\text { radius of } \\
\text { Planets (m) }\end{array}$ & $\begin{array}{c}\text { ReNFR }(m) \\
\text { using } \\
\text { equation } \\
2.1\end{array}$ & $\begin{array}{c}\mathbf{R}^{3} / \mathbf{T}^{2} \\
\left(\mathbf{m}^{3} / \mathbf{s}^{2}\right)^{1}\end{array}$ & $\begin{array}{c}\text { Wavelength } \\
\text { (m) } \\
\text { using } \\
\text { equation } 2.4\end{array}$ & $\begin{array}{c}\text { ReNFR(m) } \\
\text { Using } \\
\text { equation } 2.4\end{array}$ \\
\hline 1. & Mercury & $4.88 \mathrm{E}+06$ & $0.49 \mathrm{E}-03$ & $3.02 \mathrm{E}+11$ & $3.36 \mathrm{E}+18$ & 2953.25 & $1.23 \mathrm{E}+08$ \\
\hline 2. & Venus & $1.21 \mathrm{E}+07$ & 7.23E-03 & $3.07 \mathrm{E}+11$ & $3.36 \mathrm{E}+18$ & 2953.26 & $4.80 \mathrm{E}+08$ \\
\hline 3. & Earth & $1.27 \mathrm{E}+07$ & 8.87E-03 & $2.98 \mathrm{E}+11$ & $3.36 \mathrm{E}+18$ & 2953.15 & $5.17 \mathrm{E}+08$ \\
\hline 4. & Mars & $6.75 \mathrm{E}+06$ & $0.95 \mathrm{E}-03$ & $3.52 \mathrm{E}+11$ & $3.36 \mathrm{E}+18$ & 2952.55 & $2.00 \mathrm{E}+08$ \\
\hline 5. & Jupiter & $1.34 \mathrm{E}+08$ & 2.82 & $5.71 \mathrm{E}+11$ & $3.37 \mathrm{E}+18$ & 2958.82 & $1.76 \mathrm{E}+10$ \\
\hline 6. & Saturn & $1.09 \mathrm{E}+08$ & 0.84 & $7.65 \mathrm{E}+11$ & $3.41 \mathrm{E}+18$ & 2994.88 & $1.28 \mathrm{E}+10$ \\
\hline 7. & Uranus & $4.99 \mathrm{E}+07$ & 0.13 & $6.09 \mathrm{E}+11$ & $3.37 \mathrm{E}+18$ & 2962.24 & $4.02 \mathrm{E}+09$ \\
\hline 8. & Neptune & $4.87 \mathrm{E}+07$ & 0.15 & $5.40 \mathrm{E}+11$ & $3.36 \mathrm{E}+18$ & 2950.48 & $3.88 \mathrm{E}+09$ \\
\hline
\end{tabular}

${ }^{1} \mathrm{R}$ is semimajor axis of planets and $\mathrm{T}$ is the orbital period of planets 
Table 8 Tabulates the Sun's Reactive Near Field Radius (ReNFR) using the Schwarzschild radius, multiplied by 10 and 100 and divided by $10,100,1000$, and 10000 .

\begin{tabular}{|c|c|c|c|c|}
\hline $\begin{array}{l}\text { Features } \\
\text { of Sun }\end{array}$ & & Wavelength (m) & $\begin{array}{c}\operatorname{ReNFR}(\mathbf{m}) \\
\text { using equation } 1^{1}\end{array}$ & $\begin{array}{c}\text { ReNFR } \\
\text { (AU) }\end{array}$ \\
\hline 1. & \multirow{7}{*}{ 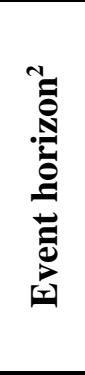 } & $100 \lambda_{s}{ }^{3}$ & $5.92 \mathrm{E}+10$ & 0.3956 \\
\hline 2. & & $10 \lambda_{s}$ & $1.87 \mathrm{E}+11$ & 1.2511 \\
\hline 3. & & $\lambda_{\mathrm{s}}$ & $5.92 \mathrm{E}+11$ & 3.9564 \\
\hline 4. & & $\lambda_{s} / 10$ & $1.87 \mathrm{E}+12$ & 12.5114 \\
\hline 5. & & $\lambda_{s} / 100$ & $5.92 \mathrm{E}+12$ & 39.5644 \\
\hline 6. & & $\lambda_{s} / 1000$ & $1.87 \mathrm{E}+13$ & 125.1136 \\
\hline 7. & & $\lambda_{s} / 10000$ & $5.92 \mathrm{E}+13$ & 395.6439 \\
\hline 8. & \multirow{7}{*}{ 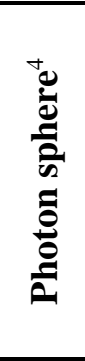 } & $100\left(\lambda_{s} \times 1.5\right)$ & $4.83 \mathrm{E}+10$ & 0.3230 \\
\hline 9. & & $10\left(\lambda_{s} \times 1.5\right)$ & $1.53 \mathrm{E}+11$ & 1.0215 \\
\hline 10. & & $\left(\lambda_{s} \times 1.5\right)$ & $4.83 \mathrm{E}+11$ & 3.2304 \\
\hline 11. & & $\left(\lambda_{s} \times 1.5\right) / 10$ & $1.53 \mathrm{E}+12$ & 10.2155 \\
\hline 12. & & $\left(\lambda_{s} \times 1.5\right) / 100$ & $4.83 \mathrm{E}+12$ & 32.3042 \\
\hline 13. & & $\left(\lambda_{s} \times 1.5\right) / 1000$ & $1.53 \mathrm{E}+13$ & 102.1548 \\
\hline 14. & & $\left(\lambda_{s} \times 1.5\right) / 10000$ & $4.83 \mathrm{E}+13$ & 323.0419 \\
\hline
\end{tabular}

\footnotetext{
${ }^{1}$ Polar diameter of the Sun $=1.39 \mathrm{E}+09 \mathrm{~m}$

${ }^{2}$ Far-field of Event Horizon is $1.31 \mathrm{E}+15 \mathrm{~m}(8758.8851 \mathrm{AU})$

${ }^{3} \lambda_{\mathrm{s}}=$ Schwarzschild Radius of Sun $=2953.38 \mathrm{~m}$

${ }^{4}$ Far-field of Photon Sphere is 8.74E+14 m (5839.2567 AU)
} 


\section{Supplementary information}

The Communication Infrastructures of Living Systems and the Universe: Analysis from the Perspective of Antenna Theory

Sirsendu Sekhar Ray, Bitop Maitra

\section{Supplementary Equations}

\subsection{Helical Antenna}

The basic structure of a helix depends upon its diameter (D), Pitch (S), and the number of turns (N).

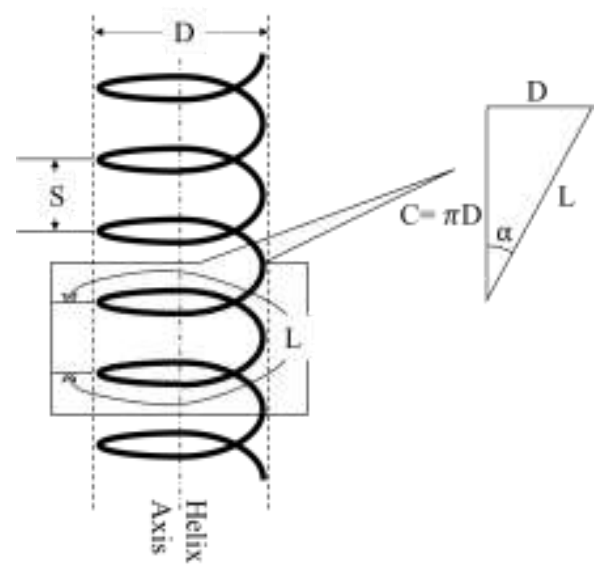

Supplementary figure 1.1 Helical antenna

\subsubsection{Axial mode wavelength}

The axial mode wavelength of the helix is approximately equal to the circumference of the helix.

The circumference $(\mathrm{C})$ of the helix is calculated as

$$
\mathrm{C}=\pi \mathrm{D}=\text { Axial mode wavelength }
$$

\subsubsection{Normal mode wavelength}

In the Normal mode, the circumference of the antenna is lesser than the wavelength. The radiation pattern is along the normal plane of the axis, and the maximum radiation occurs at that length where the wavelength of the helix is proportional to the length and nearly null at the axis. Axial Ratio (AR) is given by

$$
\mathrm{AR}=\frac{2 \mathrm{~S}_{\lambda}}{\mathrm{C}_{\lambda}^{2}} ;\left[\begin{array}{c}
\text { If } \mathrm{AR}=0 ; \text { horizontally polarised } \\
\mathrm{AR}=\infty ; \text { vertically polarised } \\
\mathrm{AR}=1 ; \text { circularly polarised }
\end{array}\right]
$$

For $\mathrm{AR}=1$, wavelength $(\lambda)$ is expressed as

$$
\lambda=\frac{\mathrm{C}^{2}}{2 \mathrm{~S}}=\text { Normal mode circularly polarized wavelength }
$$

\subsubsection{Wirelength of antenna}

Length (L) of each turn of the wire is given by

$$
\mathrm{L}=\sqrt{\mathrm{S}^{2}+\mathrm{C}^{2}}
$$




\subsubsection{Wirelength of small helix antenna}

Wirelength (W) of small helix is given by

$$
\mathrm{W}=\mathrm{S}+\mathrm{C}
$$

\subsubsection{Self-resonance wavelength}

Self-resonance wavelength is calculated by

$$
\frac{279\left(\frac{\mathrm{H}_{\mathrm{A}}}{\lambda}\right)}{\mathrm{N} \pi\left(0.92 \frac{\mathrm{H}_{\mathrm{A}}}{\lambda}+\frac{\mathrm{D}_{\mathrm{A}}}{\lambda}\right)^{2}}=\frac{600 \pi \times 19.7\left(\frac{\mathrm{D}_{\mathrm{A}}}{\lambda}\right)^{2}}{9 \frac{\mathrm{D}_{\mathrm{A}}}{\lambda}+20 \frac{\mathrm{H}_{\mathrm{A}}}{\lambda}}
$$

\subsubsection{Wavelength of half-turn bifilar antenna}

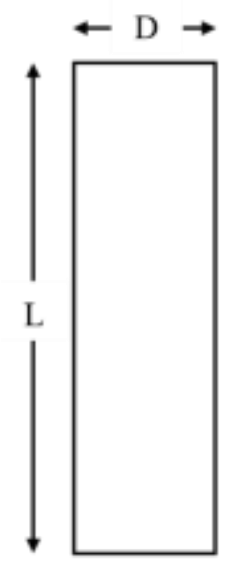

Supplementary figure 1.2 Half-turn bifilar helical antenna

Half-turn bifilar antenna wavelength is given by

$$
\lambda=2(L+D)
$$

\subsubsection{Pitch angle}

Pitch angle is calculated as

$$
\alpha=\tan ^{-1} \frac{S}{\pi D}
$$

\subsection{Zig Zag Antenna}

Zig-zag antenna is a type of traveling wave antenna.

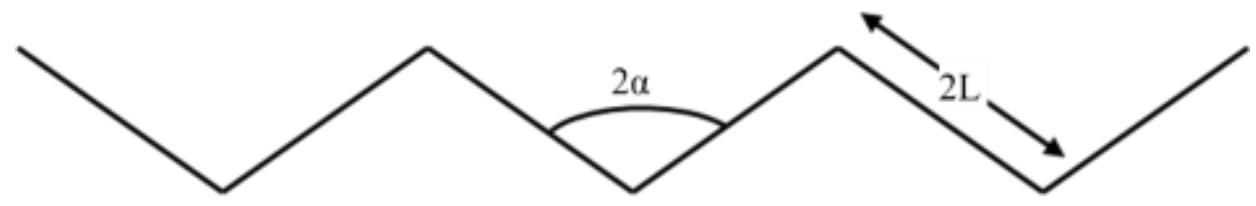

Supplementary figure 1.3 Zig-zag antenna

The relationship between the arm length (2L) and the pitch angle $(2 \alpha)$ of a zig-zag antenna is given by

$$
\frac{4 \mathrm{~L}}{\lambda}=\frac{1}{1-\sin \alpha} ;\left\{\begin{array}{c}
\mathrm{L}=\text { Arm length of the antenna, } \\
\lambda=\text { wavelength, } \\
\alpha=\text { pitch angle }
\end{array}\right.
$$

\subsection{Vee Antenna}


Vee antenna is a special type of dipole antenna where the dipoles are tilted at an angle of ' $\alpha$ ' with an arm length of 'l.' The tilt angle of the vee antenna depends upon the arm length and the wavelength $(\lambda)$.

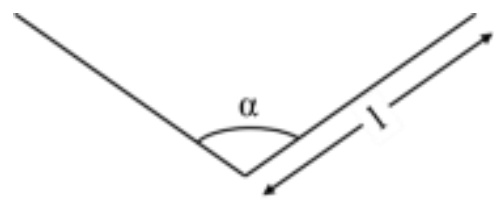

Supplementary figure 1.4 Vee antenna

This relationship among them is given by

$$
\begin{gathered}
\alpha=-149.3\left(\frac{1}{\lambda}\right)^{3}+603.4\left(\frac{1}{\lambda}\right)^{2}-809.5\left(\frac{l}{\lambda}\right)+443.6 ; \text { for } 0.5 \leq \frac{1}{\lambda} \leq 1.5 \\
\alpha=13.39\left(\frac{1}{\lambda}\right)^{2}-78.27\left(\frac{1}{\lambda}\right)+169.77 ; \text { for } 1.5 \leq \frac{1}{\lambda} \leq 3
\end{gathered}
$$

\subsection{Horn Antenna}

A horn antenna with wavelength $\lambda$ and slant length in E-field as $S_{E}$, the aperture in the E-field direction $\left(\mathrm{A}_{\mathrm{E}}\right)$ is given by

$$
\mathrm{A}_{\mathrm{E}}=\sqrt{2 \lambda \mathrm{S}_{\mathrm{E}}}
$$

The aperture in the $\mathrm{H}$-field direction $\left(\mathrm{A}_{\mathrm{H}}\right)$ with slant length $\mathrm{S}_{\mathrm{H}}$ is given by

$$
\mathrm{A}_{\mathrm{H}}=\sqrt{3 \lambda \mathrm{S}_{\mathrm{H}}}
$$

\subsection{Cut-off wavelength of waveguide}

The cut-off frequency of TE mode is:

$$
\mathrm{f}_{\mathrm{c}_{\mathrm{mn}}}^{\mathrm{TE}}=\frac{\chi_{\mathrm{mn}}^{\prime}}{2 \pi \mathrm{a} \sqrt{\mu_{0} \varepsilon_{0}}}
$$

The cut-off frequency of TM mode is:

$$
\mathrm{f}_{\mathrm{C}_{\mathrm{mn}}}^{\mathrm{TM}}=\frac{\chi_{\mathrm{mn}}}{2 \pi \mathrm{a} \sqrt{\mu_{0} \varepsilon_{0}}}
$$

$\chi_{m n}$ and $\chi_{m n}^{\prime}$ are $\mathrm{n}^{\text {th }}$ zero of the $\mathrm{m}^{\text {th }}$ order Bessel function and Bessel function derivative, respectively. $\mu_{0}$ and $\varepsilon_{0}$ are the free space permeability and free space permittivity, respectively.

The cut-off wavelength $(\lambda)$ with the velocity of light (c) is given by

$$
\lambda_{\mathrm{c}}=\frac{\mathrm{c}}{\mathrm{f}_{\mathrm{c}_{\mathrm{mn}}}}
$$

\subsection{Antenna Gain}

The gain $(\mathrm{G})$ is given by

$$
\mathrm{G}=\frac{4 \pi \mathrm{A}}{\lambda^{2}} \mathrm{E}_{\mathrm{A}}
$$

Where ' $A$ ' is the antenna aperture, ' $E_{A}$ ' is the aperture efficiency and ' $\lambda$ ' is the wavelength of the antenna. 